\title{
INT-767 prevents NASH and promotes visceral fat brown adipogenesis and mitochondrial function
}

\author{
Paolo Comeglio1,*, Ilaria Cellai1,*, Tommaso Mello², Sandra Filippi³, Elena Maneschi', Francesca Corcetto1, \\ Chiara Corno', Erica Sarchielli4, Annamaria Morelli4, Elena Rapizzi5, Daniele Bani4, Daniele Guasti4, \\ Gabriella Barbara Vannelli4, Andrea Galli2, Luciano Adorini6, Mario Maggi1,7 and Linda Vignozzi1,7,8
}

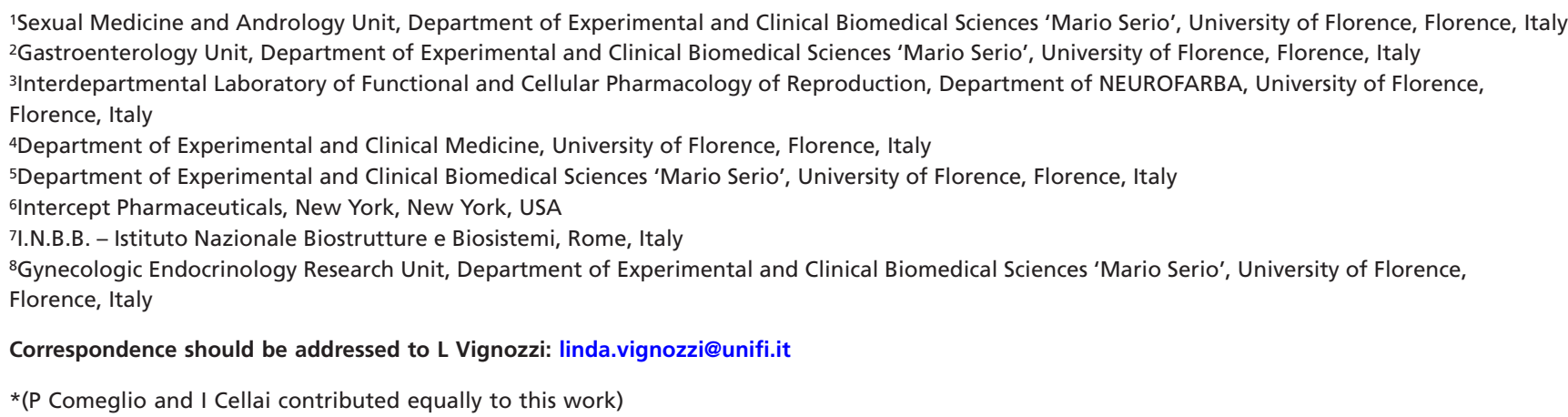

\section{Abstract}

The bile acid receptors, farnesoid X receptor (FXR) and Takeda G-protein-coupled receptor 5 (TGR5), regulate multiple pathways, including glucose and lipid metabolism. In a rabbit model of high-fat diet (HFD)-induced metabolic syndrome, long-term treatment with the dual FXR/TGR5 agonist INT-767 reduces visceral adipose tissue accumulation, hypercholesterolemia and nonalcoholic steatohepatitis. INT-767 significantly improves the hallmarks of insulin resistance in visceral adipose tissue (VAT) and induces mitochondrial and brown fat-specific markers. VAT preadipocytes isolated from INT-767-treated rabbits, compared to preadipocytes from HFD, show increased mRNA expression of brown adipogenesis markers. In addition, INT-767 induces improved mitochondrial ultrastructure and dynamic, reduced superoxide production and improved insulin signaling and lipid handling in preadipocytes. Both in vivo and in vitro treatments with INT-767 counteract, in preadipocytes, the HFD-induced alterations by upregulating genes related to mitochondrial biogenesis and function. In preadipocytes, INT-767 behaves mainly as a TGR5 agonist, directly activating dose dependently the CAMP/PKA pathway. However, in vitro experiments also suggest that FXR activation by INT-767 contributes to the insulin signaling improvement. INT-767 treatment counteracts HFD-induced liver histological alterations and normalizes the increased pro-inflammatory genes. INT-767 also induces a significant reduction of fatty acid synthesis and fibrosis markers, while increasing lipid handling, insulin signaling and mitochondrial markers. In conclusion, INT-767 significantly counteracts HFD-induced liver and fat alterations, restoring insulin sensitivity and prompting preadipocytes differentiation toward a metabolically healthy phenotype.

\section{Key Words}

- insulin resistance

- mitochondrial metabolism

- preadipocytes

- metabolic syndrome

- INT-767 


\section{Introduction}

Metabolic syndrome (MetS) is associated with a wide range of cardiometabolic risk conditions, including coronary heart disease, type 2 diabetes mellitus and nonalcoholic fatty liver disease (NAFLD) (Corona et al. 2007). Among them, insulin resistance and visceral adiposity play a central role. In MetS, adipocytes become severely dysfunctional and insulin resistant, showing a reduced metabolic capacity to store surplus energy (Maneschi et al. 2016), eventually leading to fat deposition and inflammation within other tissues that regulate metabolic homeostasis, such as the liver. Nonalcoholic steatohepatitis (NASH) is considered the hepatic hallmark of insulin resistance associated to MetS and obesity, and NASH development is related to increased size and volume of adipocytes, which are closely related to insulin sensitivity (Bedossa et al. 2016).

Bile acids (BAs) are hormone-like signaling molecules that regulate glucose and lipid metabolism as well as energy homeostasis (Houten et al. 2006, Lefebvre et al. 2009). The family of BA-activated receptors (BARs) includes the Takeda G-protein-coupled receptor 5 (TGR5) and the nuclear farnesoid X receptor (FXR). Upon activation, TGR5 leads to rapid stimulation of the cAMP-dependent pathway, with accumulation of cAMP and activation of protein kinase $\mathrm{A}$ (PKA). In the intestine, BAs released after food ingestion activate TGR5 in enteroendocrine L-cells, leading to secretion of glucagon-like peptide-1 (GLP-1), which further stimulates postprandial insulin secretion from pancreas. In high-fat diet (HFD) animal models, treatment with a specific TGR5 agonist, INT-777 (Pellicciari et al. 2009), improved glucose tolerance (Thomas et al. 2009) and substantially reduced visceral adiposity (Maneschi et al. 2013). These beneficial effects seem to occur partially by enhancing energy expenditure through activation of thermogenesis in brown adipose tissue (BAT) and in muscle (Thomas et al. 2009). Activation of cAMP/PKA signaling by TGR5 induces cAMP-responsive element-binding protein 1 (CREB1), leading to increased type II iodothyronine deiodinase (DIO2), which activates the precursor thyroid hormone, thyroxine, thereby resulting in increased BAT activity and energy expenditure (Thomas et al. 2008).

FXR belongs to the family of nuclear receptors and is predominantly expressed in the liver, kidney and intestine, with a major role in controlling BA homeostasis. Through the induction of small heterodimer partner (SHP) in the liver and fibroblast growth factor 19 (FGF19) (FGF15 in rodents) in the intestine, FXR activation leads to inhibition of cholesterol $7 \alpha$-hydroxylase (CYP7A1), the rate-limiting enzyme in hepatic BA synthesis. In addition to BA homeostasis, FXR regulates lipid and glucose metabolism (Zhang et al. 2006). Obeticholic acid (OCA) is a first-in-class potent and selective FXR agonist. We have previously investigated the effects of OCA in a rabbit non-genomic model of MetS induced by long-term administration of a HFD (Morelli et al. 2012, Maneschi et al. 2013, Vignozzi et al. 2014). This model recapitulates the features of human MetS, including hypertension, hyperglycemia, dyslipidemia, reduced glucose tolerance, visceral fat accumulation and NASH (Morelli et al. 2012, Maneschi et al. 2013, 2016, Vignozzi et al. 2014). In this model, OCA administration prevents HFD-induced hyperglycemia, glucose intolerance and liver alterations, as well as visceral adipose tissue (VAT) expansion and dysfunction (Maneschi et al. 2013, Vignozzi et al. 2014). Interestingly, OCA improves MetS and NASH features not only in preclinical models (Adorini et al. 2012), but also in patients, enhancing insulin sensitivity in diabetics with NAFLD (Mudaliar et al. 2013) and ameliorating liver histology and function in non-cirrhotic NASH patients (Neuschwander-Tetri et al. 2015).

Considering the pivotal role of FXR and TGR5 in metabolic control, the aim of the present study was to investigate the effect of a dual FXR/TGR5 agonist, INT-767, on liver, metabolic parameters and VAT dysfunction in the rabbit model of MetS. Several beneficial effects of the selective TGR5 agonist, INT-777 and the selective FXR agonist, OCA, have been already demonstrated in experimental models of MetS (Thomas et al. 2009, Maneschi et al. 2013, Vignozzi et al. 2014). Therefore, we decided to use OCA and INT-777 treatments as comparators, thus helping to discriminate between FXR- and TGR5-mediated pathways induced by INT-767.

The results show that INT-767 treatment improves several MetS features, including insulin resistance, NASH and visceral adipose tissue dysfunction, remodeling visceral fat toward brown phenotype and improving mitochondrial function.

\section{Materials and methods}

\section{MetS rabbit model}

Male New Zealand White rabbits (Charles River, Calco, Lecco, Italy), age (16-week old at the beginning of treatments) and weight matched, were individually caged under standard conditions in a temperature and 
humidity controlled room on a 12-h light/darkness cycle. After 1 week of regular diet, the rabbits were randomly assigned to two different groups: untreated $(n=20)$, fed a regular diet $(\mathrm{RD})$ and treated $(n=21)$, fed a HFD, as previously described (Maneschi et al. 2016). Subgroups of HFD rabbits were treated with the FXR agonist OCA $(10 \mathrm{mg} / \mathrm{kg}$ daily, 5 days a week for 12 weeks, by oral gavage; $n=9)$, with the selective TGR5 agonist INT-777 $(30 \mathrm{mg} / \mathrm{kg}$ daily, 5 days a week for 12 weeks, by oral gavage; $n=6$ ) or with the dual FXR/TGR5 agonist INT-767 (3 mg/kg daily, 5 days a week for 12 weeks, by oral gavage; $n=8$ ). The OCA dose used has been selected based on efficacy and pharmacokinetics analysis in rodents (Rizzo et al. 2010) and has been previously utilized in rabbits (Maneschi et al. 2013). Similarly, INT-777 dose has been already reported in the literature in several experimental models (Pellicciari et al. 2009, Thomas et al. 2009, Maneschi et al. 2013). Although a dose of $30 \mathrm{mg} / \mathrm{kg} / \mathrm{die}$ of INT-767 has been reported in mice (Pathak et al. 2017, Wang et al. 2017), the administered dose of INT-767 was three-fold lower compared to OCA, to account for its three-fold higher potency in FXR activation (Rizzo et al. 2010). All compounds were provided by Intercept Pharmaceuticals Inc. (New York, NY, USA). Mean arterial pressure (MAP) and oral glucose tolerance test (OGTT) was measured before killing, as previously described (Filippi et al. 2009). This study was carried out in strict accordance with the Italian Ministerial Law \#116/92 for the Care and Use of Laboratory Animals. The protocol was approved by the Institutional Animal Care and Use Committee of the University of Florence. After killing, liver, small intestine and visceral fat were harvested from the different experimental groups and stored for the subsequent analyses. Blood samples for glucose, total cholesterol and triglycerides and transaminases (AST and ALT) analyses were obtained from the marginal ear vein at week 12 , in all groups.

\section{Histomorphometric analysis and hypoxia detection in VAT}

Immunohistochemical localization of FXR and TGR5 was performed on deparaffinized rehydrated sections as previously described (Vignozzi et al. 2017).

To determine the diameter of adipocytes, sectional areas of VAT in hematoxylin and eosin-stained preparations were analyzed as previously described (Maneschi et al. 2012). VAT specimens were analyzed by bio-reductive drug pimonidazole hydrochloride (hypoxyprobe, $1.60 \mathrm{mg} / \mathrm{kg}$ ), to measure hypoxia, as previously described (Maneschi et al. 2012).

\section{Liver histomorphological analysis}

Liver specimens were analyzed by bio-reductive drug pimonidazole hydrochloride (hypoxyprobe, $1.60 \mathrm{mg} / \mathrm{kg}$ ), to measure hypoxia, as previously described (Maneschi et al. 2012). To evaluate lipid accumulation, frozen liver sections were cut in a cryostat and stained with Oil Red-O for $20 \mathrm{~min}$, as previously described (Maneschi et al. 2013). Collagen content evaluation was blindly quantified by Picrosirius Red Assay (Bio-Optica, Milan, Italy) staining. Sections were fixed in $10 \%$ buffered-formalin, embedded in paraffin, then sectioned at a thickness of $5 \mu \mathrm{m}$ and stained per the manufacturer's instructions, as previously described (Comeglio et al. 2017). Sectional areas of liver in hematoxylin and eosin-stained preparations were analyzed as previously described (Maneschi et al. 2012). Immunohistochemical studies for interferon regulatory factor 5 (IRF5) localization were performed on deparaffinized rehydrated sections. Briefly, deparaffinized and rehydrated sections were incubated overnight at $4^{\circ} \mathrm{C}$ with mouse monoclonal IRF5 primary antibody (1:50 vol/vol; Santa Cruz Biotechnology), and then rinsed in PBS, incubated with the biotinylated secondary goat anti-mouse antibody (Millipore Corporation) and then with a streptavidin-biotin peroxidase complex (Lab-Vision, Fremont, CA, USA). The reaction product was developed with 3,3'-diaminobenzidine tetrahydrochloride as chromogen (Sigma-Aldrich). The slides were evaluated and photographed using a Nikon Microphot-FXA microscope (Nikon).

\section{Preparation of total and membrane/cytosolic fractions for Western blot analysis}

Samples of VAT and liver were immediately frozen in liquid nitrogen after isolation. Membrane and cytosolic protein fractions were prepared using the ProteoExtract sub-cellular proteome extraction kit (Calbiochem, EMD Biosciences), per the manufacturer's instructions. Western blot analysis with an anti-glucose transporter type 4 (GLUT4) antibody (1:500 vol/vol; Upstate Biotechnology, Lake Placid, NY, USA), anti-ras homolog member A (RhoA) (1:500 vol/vol; Santa Cruz Biotechnology) and anti-theromogenin (UCP1) antibody (1:200 vol/vol; Santa Cruz Biotechnology) was performed as previously described (Maneschi et al. 2016). 
Isolation, characterization and differentiation of rabbit visceral fat preadipocytes

Rabbit visceral fat preadipocytes (rPAD) isolation and characterization from VAT samples was performed as previously described (Maneschi et al. 2016). The spontaneous adipogenic potential was investigated in rPADs cultured for 10 days in 5\% FBS-supplemented DMEM. The rPADs differentiation, 2 days after confluence (time 0 ), was induced by exposing cells to a differentiation mixture (DIM) containing $5 \mathrm{mg} / \mathrm{mL}$ insulin, $1 \mathrm{mM}$ dexamethasone (Sigma-Aldrich) and 0.5 mM 3-isobutyl1-methylxanthine (IBMX; Sigma-Aldrich) in 5\% stripped FBS-supplemented DMEM for 8 days (Student et al. 1980, Maneschi et al. 2016). Glucose uptake in DIM-rPADs, alone or stimulated for 10 days with INT-767 $1 \mu \mathrm{M}$, was performed as previously described (Maneschi et al. 2012).

\section{Fluorescence and electronic microscopy}

Untreated and DIM-induced rPADs, isolated from each experimental group, were cultured on $35 \mathrm{~mm}$ u-Dish (Ibidi, Munich, Germany) for 10 days and then stained with $200 \mathrm{nM}$ MitoTracker Green (Invitrogen), alone or in combination with $10 \mu \mathrm{M}$ dihydroethidium (DHE; Invitrogen) or AdipoRed Assay (Cambrex BioScience, Walkersville, MD, USA), as previously described (Maneschi et al. 2016).

For mitochondrial morphometric analysis, regions of interest in the periphery of cells were considered, where individual mitochondria are readily resolved, and the mitochondrial length was measured. Time lapses were recorded for $3 \mathrm{~min}$ with $10 \mathrm{~s}$ time intervals. DHE fluorescence was used to measured superoxide radical production in untreated rPADs. The superoxide radical production was quantified using Fiji ImageJ software (Schindelin et al. 2012) by measuring the change in fluorescence intensity in the nuclei of rPAD cells during imaging, as previously described (Maneschi et al. 2016).

To detect qualitative accumulation of intracellular lipids, cells were washed in PBS, fixed in 10\% bufferedformalin for $30 \mathrm{~min}$ at room temperature (RT), stained with AdipoRed (prepared according to manufacturer's instructions) and DAPI ( $1 \mu \mathrm{g} / \mathrm{mL}$; Roche) at RT for $10 \mathrm{~min}$ and mounted with Prolong Gold anti-fade reagent (Life Technologies). Images were acquired with a DM6000 microscope (Leica) equipped with a DFC350FX camera, using a 10x/0.3na HC PL Fluotar objective and L5/A4 Leica filter set for AdipoRed and DAPI, respectively. AdipoRedpositive cells, identified as those clearly showing lipid droplets staining, and total cells (number of nuclei) were counted. Results are showed as percentage of AdipoRedpositive cells.

For lipid droplet analysis, Z-stack images were captured sampling in accordance to Nyquist Criterion. Images were first deconvolved using Huygens Professional Software (Scientific Volume Imaging, Hilversum, The Netherlands) using the Classic Maximum Likelihood Estimation algorithm and a theoretical Point Spread Function. Deconvolved images were then quantitatively analyzed using the Volocity 5 Software (Perkin-Elmer) to measure the volume and number of lipid droplets.

Electronic microscopy was used in untreated rPADs cultured for 10 days in 5\% FBS-supplemented DMEM, then pelleted by centrifugation, fixed in Karnowsky buffer and 1\% osmium tetroxide and embedded in Epon 812 (EMS, Hatfield, PA, USA). Mitochondrial and internal cristae surface area in stained ultrathin sections were measured using iTEM image analysis software (EMSIS, Muenster, Germany), as previously described (Maneschi et al. 2016).

Immunocytochemical detection of UCP-1 was carried out on rPADs isolated from HFD rabbits, alone or treated with INT-767 $1 \mu \mathrm{M}$ (or with an equimolar dose of the two selective TGR5 and FXR agonist, INT-777 and OCA), cultured on slides for 10 days. Cells were then fixed in $2 \%$ paraformaldehyde in PBS for $10 \mathrm{~min}$ at RT, followed by permeabilization with $0.1 \%$ Triton X-100 in PBS for $10 \mathrm{~min}$ and blocking with $1 \%$ BSA in PBS. Immunostaining was performed with anti-UCP-1 goat polyclonal antibody (1:50 vol/vol, Santa Cruz Biotechnology), followed by Alexa Fluor 488 donkey anti-goat IgG $(\mathrm{H}+\mathrm{L})(1: 200 \mathrm{vol} / \mathrm{vol}$, Molecular Probes). Antibody specificity was verified by omitting the primary antibody. The percentage of UCP-1-positive cells was calculated by counting the stained cells in ten fields per slide of three different experiments normalized on total cells (DAPI stained).

\section{Oxygen consumption and intracellular ATP analysis}

Quantification of oxygen consumption by rPADs isolated from each experimental group was conducted by means of the Oxygraph system (Hansatech Instruments, Pentney, UK), as previously described (Rapizzi et al. 2015). Briefly, cells $\left(7.5 \times 10^{4}\right)$ were loaded in the chamber, which contained $300 \mu \mathrm{L}$ of DMEM with glutamine $2 \mathrm{mM}$ and sodium succinate $20 \mathrm{mM}$. Oxygen consumption was monitored for $5 \mathrm{~min}$ at $37^{\circ} \mathrm{C}$.

ATP levels were measured using the CellTiter-Glo luminescent cell viability assay (Promega Corporation). This assay was performed according to the manufacturer's 
protocol. Briefly, rPADs isolated from each experimental group were seeded into 96 -well plates $\left(2 \times 10^{4}\right.$ cells/well $)$ and cultured in complete growth medium. To evaluate the effect of spontaneous differentiation, cells were stimulated for 10 days in 5\% stripped FBS-supplemented DMEM in the presence or not of INT-767 $1 \mu \mathrm{M}$, and then incubated with $125 \mu \mathrm{L}$ of CellTiter-Glo reagent. The cultures were shaken at $300 \mathrm{rpm}$ for $5 \mathrm{~min}$ and then incubated at RT for $25 \mathrm{~min}$ to stabilize the luminescent signal. Luminescence was measured using the VICTOR ${ }^{3}$ 1420 Multilabel Counter (Packard Instruments, PerkinElmer) and normalized to number of cells.

\section{$\mathrm{rPAD}_{\mathrm{s}}$ in vitro experiments}

rPADs were plated $\left(8 \times 10^{4}\right.$ cells/well $)$ and cultured in complete growth medium until confluence, then they were stimulated for 10 days in 5\% stripped FBS-supplemented DMEM with INT-767 $1 \mu \mathrm{M}$ or with an equimolar dose of the two selective TGR5 and FXR agonist, INT-777 and OCA. To further investigate the role of TGR5 activation, rPADs were stimulated for $18 \mathrm{~h}$ with INT-767 $(1 \mu \mathrm{M})$, alone or with cell-permeable protein kinase A inhibitor (PKI, $10 \mu \mathrm{M}$; Merck) or forskolin ( $10 \mu \mathrm{M}$; Sigma-Aldrich), a direct activator of adenylate cyclase (Insel \& Ostrom 2003).

\section{Quantitative determination of intracellular cAMP}

Intracellular cyclic AMP was measured using a cAMP competitive ELISA kit according to the manufacturer's instructions (Invitrogen). Briefly, rPADs isolated from HFD experimental group were plated $\left(5 \times 10^{4}\right.$ cells/well $)$ and maintained in DMEM supplemented with 10\% FBS until the confluence. Cells were then washed and transferred to serum-free medium for $24 \mathrm{~h}$. Subsequently, cells were treated with increasing concentrations $(0.3-30 \mu \mathrm{M})$ of INT-767 for $1 \mathrm{~min}$, culture media was removed and cells were lysed with $0.1 \mathrm{M} \mathrm{HCl}$, to stop endogenous phosphodiesterase activity, and after centrifugation, the supernatants were used directly in the assay.

\section{Isolation, characterization and siRNA analysis of human visceral fat preadipocytes}

Adipose tissue samples were obtained, following informed patient consent, from eight subjects (obese males, age $25-65$ years; $B M I \geq 40 \mathrm{~kg} / \mathrm{m}^{2}$ ) undergoing bariatric surgery for weight loss. All subjects affected by cancer, infections, chronic or acute inflammation and autoimmune diseases were excluded. The protocol used in the present study was reviewed and approved by the Institutional Review Board of the Florence University Hospital.

Human visceral fat preadipocytes (hPAD) isolation and characterization from VAT samples was performed from adipose tissue biopsies obtained by bariatric surgery as previously reported (Zuk et al. 2001, Baglioni et al. 2009). A confluent and homogeneous fibroblast-like cell population (hPADs) was obtained within 2-3 weeks. Only cells at early culture passages were used, and each experiment was repeated at least three times.

Cell immunophenotypical analysis of cultured hPADs was performed using the FITC-, PE-or APC-conjugated monoclonal antibodies (mAbs) against CD14, CD31, CD34, CD44, CD45, CD73, CD90, CD105, CD166 and histocompatible locus antigen-I and -II (HLA-I and HLA-DR), and their respective isotype control mAbs (BD Biosciences, Mountain View, CA, USA) as previously described (Krampera et al. 2003).

Flow cytometric analysis of the surface markers on cultured hPADs isolated from different subjects showed a similar immunophenotypic profile (Zannettino et al. 2008), characterized by a marked positivity for stromal mesenchymal stem cell markers (CD44, CD73, CD90, CD105, CD166); moreover, they were negative for endothelial (CD31), hematopoietic (CD34 and CD45) and monocytic (CD14) markers (data not shown).

\section{RNA extraction, quantitative RT-PCR analysis and siRNA analysis}

Isolation of total RNA from tissues and cells, cDNA synthesis and quantitative real-time RT-PCR were performed as previously described (Comeglio et al. 2017). Specific PCR primers for rabbit target genes were designed on sequences available at NCBI GenBank (http://www.ncbi.nlm.nih. gov) or Ensemble Genome (http://www.ensembl.org) and were purchased from Life Technologies. In particular, rabbit FGF19 sequence (ENSOCUT00000003867.3) was used as template. FGF15 and FGF19 are orthologous proteins with similar functions, with the former described in the mouse and the latter found in the rabbit (Shang et al. 2014). The $18 \mathrm{~S}$ ribosomal RNA subunit was used as the reference gene for the relative quantization of the target genes based on the comparative threshold cycle (Ct) 2- $\Delta \Delta \mathrm{Ct}$ method (Livak \& Schmittgen 2001).

siRNA targeting human FXR (NR1H4 ON-TARGETplus SMARTpool) and TGR5 (GPBAR1 ON-TARGETplus SMARTpool), positive (ON-TARGETplus GAPD Control Pool) and negative (ON-TARGETplus Non-Targeting Pool) sequences were purchased from Dharmacon (Lafayette, CO, 
USA). Cells were treated with OCA, INT-767 and INT-777 $(1 \mu \mathrm{M})$ for $72 \mathrm{~h}$. Transfection was performed in subconfluent hPADs for $72 \mathrm{~h}$ with $25 \mathrm{nM}$ of siRNA by DharmaFECT transfection reagent (Dharmacon) in DMEM-F12 medium, according to the manufacturer's instruction. The efficiency of knockdown was measured by qRT-PCR (GAPD, FXR and TGR5). Quantitative mRNA expression of target genes was performed as described above.

\section{Statistical analysis}

Results are expressed as mean \pm s.E.M. For mitochondria length, median with minimum and maximum values are reported. The statistical analysis was performed with a one-way ANOVA test followed by Mann-Whitney post hoc analysis to evaluate the differences between groups, with $P<0.05$ as significant. Statistical analysis was performed with the SPSS 24.0 (SPSS). Half-maximal response effective concentration $\left(\mathrm{EC}_{50}\right)$ values and maximal effect $\left(E_{\max }\right)$ values were calculated using the computer program ALLFIT (DeLean et al. 1978).

\section{Results}

\section{FXR and TGR5 expression in rabbit tissues}

FXR and TGR5 mRNA expression was examined in a large panel of rabbit tissues (Fig. 1). Data are expressed as fold increase relative to mRNA expression in the prostate, arbitrarily chosen as reference tissue due to the extremely low levels of both targets. The highest expression level of $F X R$ was found in intestine, followed by liver. Compared to the intestine, FXR expression showed a two-log unit lower level in (VAT) (Fig. 1A). TGR5 mRNA expression showed a relatively more homogeneous pattern with minor variations among tissues (Fig. 1B). Intestine showed the highest expression, while prostate and skeletal muscle showed the lowest one.

Feeding a HFD for 12 weeks was associated with a significant increase of both FXR and TGR5 mRNA expression in the liver, compared to rabbits fed $\mathrm{RD}$ $(P<0.001$; Fig. 1C and D, respectively). No significant modifications in receptors expression were observed in the other tissues investigated (Fig. 1C and D).

\section{Beneficial metabolic effects of INT-767 treatment in HFD-induced MetS model}

To test the in vivo effects of INT-767 on glucose and lipid metabolism, HFD rabbits were treated with INT-767.
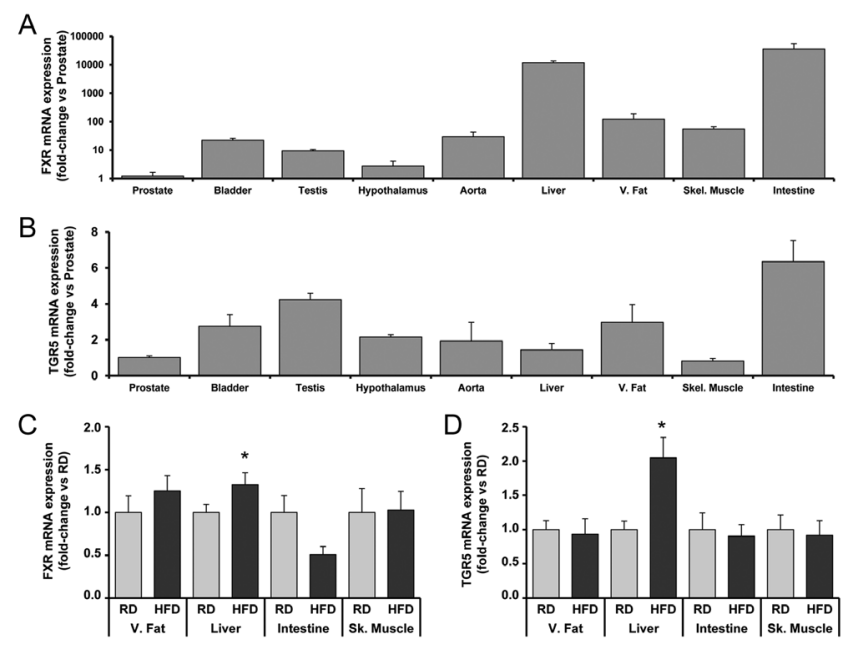

Figure 1

FXR and TGR5 expression in rabbit tissues. FXR (A) and TGR5 (B) mRNA expression were analyzed by quantitative RT-PCR in a panel of rabbit tissues isolated from RD group. Target tissue comparison in RD and HFD of FXR and TGR5 mRNA expression are reported in $C$ and $D$, respectively. Data are calculated per the $2^{-\Delta \Delta c t}$ comparative method, using the $18 \mathrm{~S}$ ribosomal RNA subunit as the reference gene for normalization. Results are expressed as fold-change of mRNA expression and reported as the mean \pm S.E.M. Statistical analysis was performed using Mann-Whitney tests $\left({ }^{*} P<0.05\right.$ vs RD).

Results were compared to those obtained in a cohort of age- and weight-matched RD rabbits and in two other cohorts of HFD rabbits treated with the FXR selective agonist, OCA, or with the TGR5 selective agonist, INT-777. Total body weight did not show any significant difference among groups (Table 1). The observed tendency in HFD body weight reduction, when compared to $\mathrm{RD}$, is reflected by a lower physical endurance, measured as the distance covered on a treadmill (RD: $241.11 \pm 107.29 \mathrm{~m} /$ running session; HFD: $116.36 \pm 64.27 \mathrm{~m} /$ running session; $P<0.01$, $n=9$ for each group). In contrast, all agonists significantly reduced VAT mass compared to HFD, even when expressed as percentage of body weight (Fig. 2A and Table 1). HFD induced a significant increase of transaminases as compared to RD (Table 1). Both transaminases showed a trend toward a reduction in all treatment groups as compared to HFD, although not reaching statistical significance (Table 1). However, all the treatments were able to reduce the hepatic-specific transaminase, ALT, to a level that was no longer significantly different when compared to RD (Table 1). In addition, INT-767 and OCA, but not INT-777, significantly ameliorated several other MetS-related parameters, such as hyperglycemia (Fig. 2B), glucose intolerance (Fig. 2C) and hypercholesterolemia (Fig. 2D). In contrast, HFD-induced increase in MAP and triglycerides levels were not significantly affected by any treatment (Fig. 2E and F, respectively). 
Table 1 Metabolic and biochemical parameters in the different rabbit experimental groups.

\begin{tabular}{|c|c|c|c|c|c|}
\hline & RD & HFD & HFD + OCA & HFD + INT-767 & HFD + INT-777 \\
\hline $\begin{array}{l}\text { Body weight (g) } \\
\text { Week } 12\end{array}$ & $3700.58 \pm 48.23$ & $3683.63 \pm 54.30$ & $3570.42 \pm 98.91$ & $3389.50 \pm 145.07$ & $3443.60 \pm 166.86$ \\
\hline $\begin{array}{l}\text { VAT }(g) \\
\text { Week } 12\end{array}$ & $29.44 \pm 1.67$ & $43.00 \pm 1.70^{\circ 00}$ & $17.00 \pm 2.53^{\circ 00, *}$ & $23.71 \pm 3.97$ * & $17.88 \pm 4.07^{\circ}, *$ \\
\hline $\begin{array}{l}\text { AST (U/L) } \\
\text { Week } 12\end{array}$ & $30.56 \pm 1.27$ & $91.33 \pm 15.08^{\circ 00}$ & $67.21 \pm 8.98^{\circ 00}$ & $61.83 \pm 5.83^{\circ 00}$ & $62.40 \pm 6.24^{\circ \circ}$ \\
\hline $\begin{array}{l}\text { ALT (U/L) } \\
\text { Week } 12\end{array}$ & $32.50 \pm 1.54$ & $55.09 \pm 3.25^{\circ 00}$ & $41.79 \pm 5.53$ & $46.75 \pm 6.50$ & $38.80 \pm 7.96$ \\
\hline
\end{tabular}

Data are expressed as mean \pm S.E.M. Data reported in bold are those that resulted significantly different among groups at the ANOVA analysis $(P<0.001)$. For these parameters, Mann-Whitney analysis was then performed $\left({ }^{\circ} P<0.05,{ }^{\circ} P<0.01,{ }^{\circ{ }^{\circ} P} P<0.001\right.$ vs RD; ${ }^{*} P<0.001$ vs HFD).

\section{INT-767 acts as a dual FXR/TGR5 agonist in liver, VAT and intestine}

We first evaluated the potential agonistic ability of INT-767 on TGR5 and FXR in tissues expressing high BAR levels, compared to the selective agonists. In the liver, FXRdependent genes - including SHP, bile salt export pump and the two hepatic FGF19 receptors (fibroblast growth factor receptor 4, FGFR4; Klothoß, KLB) - were all upregulated by INT-767, which also decreased CYP7A1 mRNA expression (Fig. 3A). INT-767 significantly induced several other FXRdependent genes, including FGF19 as well as transporters of phospholipids (multidrug resistance protein 3, MDR2; multidrug resistance-associated protein 2, MRP2) and of BAs (organic solute transporter alpha and beta, OSTA and OSTB) (Fig. 3A). These effects were also observed in OCA-treated rabbits (Fig. 3A). In contrast, INT-777 did not significantly modulate any FXR-dependent gene tested (Fig. 3A). INT-767 also stimulated the hepatic expression of genes downstream TGR5, like exchange factor directly activated by cAMP 1 (EPAC1) and PKA (Fig. 3A). A similar effect was also observed in INT-777-treated rabbits (Fig. 3A).

In VAT, INT-767 induced expression of genes related to FXR signaling (SHP) and to the TGR5 pathway (cAMPresponsive element binding protein 1, CREB1; cyclin D1, CCND1). These effects were also mimicked by OCA and INT-777 treatment, respectively (Fig. 3B).

Finally, in the small intestine, INT-767 significantly induced mRNA expression of SHP, as well as FGF19 (Fig. 3C). These effects were mimicked by FXR agonist OCA, but not by INT-777. INT-767 and INT-777 increased mRNA expression of the TGR5-dependent prohormone convertase 1 (PCSK1). A PCSK1 increase was also observed in OCA-treated rabbits.

\section{In vivo treatment with INT-767 counteracts HFD-induced VAT remodeling}

Immunohistochemical analysis showed FXR nuclear positivity in both adipocytes and endothelial cells lining

\begin{tabular}{|lr}
\hline http://joe.endocrinology-journals.org & @ 2018 Society for Endocrinology \\
https://doi.org/10.1530/JOE-17-0557 & Published by Bioscientifica Ltd. \\
Printed in Great Britain
\end{tabular}

the blood vessels (Supplementary Fig. 1, panel a and b, see section on supplementary data given at the end of this article). Likewise, TGR5 positivity was observed in the membrane of adipocytes and stromal cells (Supplementary Fig. 1, panel c and d).
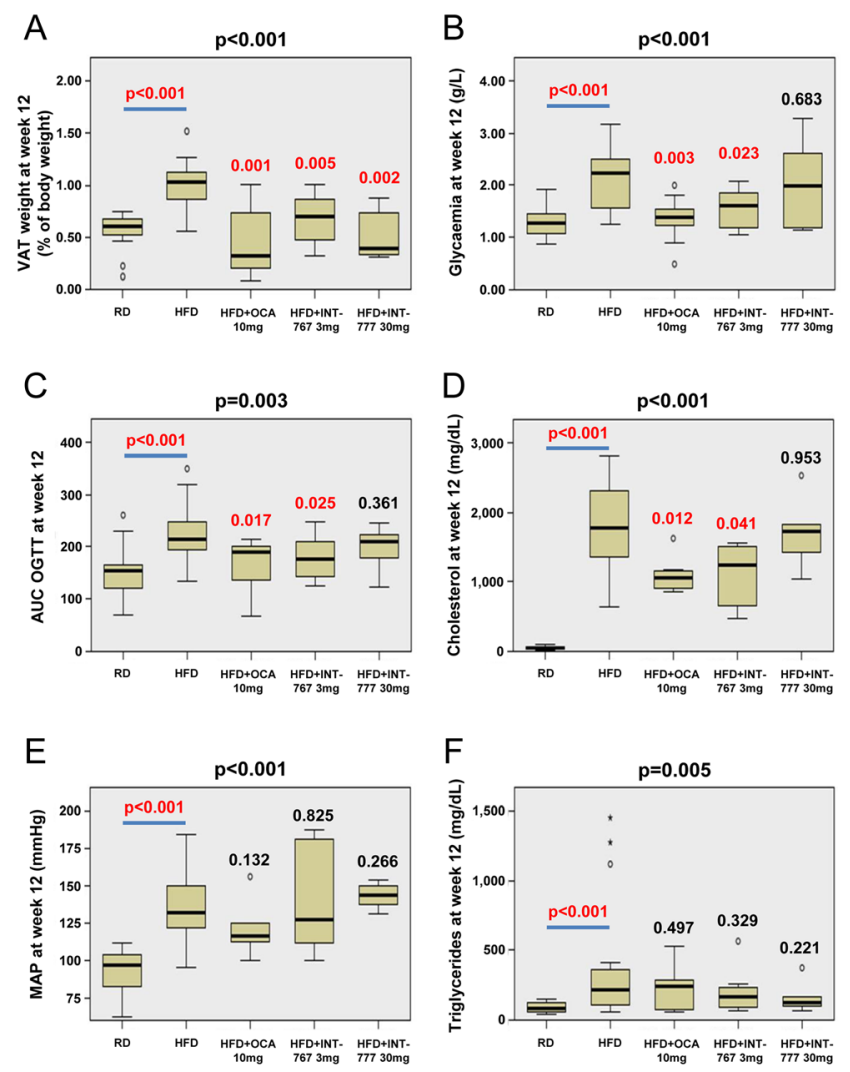

\section{Figure 2}

Metabolic effects of OCA, INT-767 and INT-777 treatments in HFD-induced MetS model. Metabolic parameters at the end of treatment (12 weeks) are reported as mean \pm S.E.M. for VAT (A), glycaemia (B), OGTT (C), Cholesterol (D), MAP (E) and triglycerides (F). Kruskal-Wallis ( $P$ values reported outside the panels) or Mann-Whitney ( $P$ values of HFD vs RD and HFD vs treatments inside the panels) analyses are reported. AUC, incremental area under the curve of glucose blood level during oral glucose tolerance test (OGTT); MAP, mean arterial pressure; VAT, visceral adipose tissue. A full colour version of this figure can be found at https:// doi.org/10.1530/JOE-17-0557. 

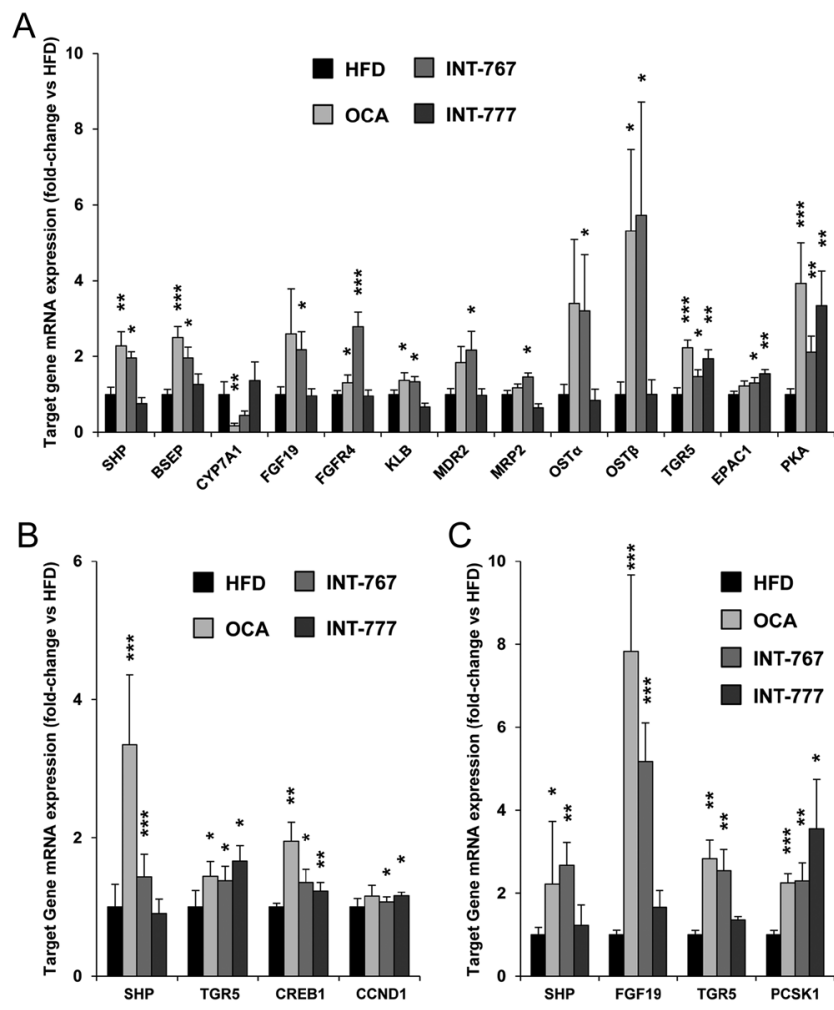

Figure 3

Comparison of OCA, INT-767 and INT-777 treatments on mRNA expression of FXR- and TGR5-related genes in rabbit liver (A), visceral fat (B) and small intestine (C). Data are calculated per the $2^{-\Delta \Delta C t}$ comparative method, using the $18 \mathrm{~S}$ ribosomal RNA subunit as reference gene for normalization and are reported as fold-change of mRNA expression over HFD. Statistical analysis was performed using the Mann-Whitney test ( $P<0.05, * * P<0.01, * * * P<0.001$ vs HFD).

Considering the relatively high mRNA expression levels of both FXR and TGR5 in VAT (Fig. 1A and B) and the ability of INT-767 to reduce VAT mass (Fig. 2A), we first investigated the effects of INT-767 in counteracting HFD-induced VAT alterations. In HFD rabbits, histomorphometric analysis of VAT specimens revealed increased adipocyte size (Fig. 4A) and decreased tissue oxygenation (Fig. 4B; both $P<0.0001$ vs RD). INT-767 treatment normalized adipocyte diameter and HFD-induced VAT hypoxia (Fig. 4A and B, respectively; both $P<0.0001)$. The intracellular localization of the insulinregulated glucose transporter GLUT4 and of the small GTP-binding protein RhoA, involved in insulin signaling, were assessed by Western blot analysis (Fig. 4C and D, respectively). In VAT extracts, HFD was associated with reduced GLUT4 (Fig. 4C; $P<0.05$ ) and with increased RhoA membrane docking (Fig. 4D; $P<0.0001$ ). Both alterations were normalized by INT-767 treatment (Fig. 4C and D). Interestingly, INT-767 treatment also significantly increased UCP1 expression in VAT, compared to HFD (Fig. 4E).
Kruskal-Wallis analysis and Mann-Whitney pairwise comparisons indicated that INT-767 significantly increased VAT mRNA expression of genes related to cell survival (B-cell lymphoma 2 (BCL2)), cGMP signaling (protein kinase G1 (PKG1)), brown adipogenesis (cell death activator CIDE-A, CIDEA; LIM homeobox 8, LHX8), mitochondriogenesis (nuclear respiratory factor 1, NRF1; peroxisome proliferator-activated receptor gamma coactivator 1-alpha, PGC1A; mitochondrial transcription factor A, TFAM), insulin signaling (six transmembrane protein of prostate 2, STAMP2), lipid metabolism (peroxisome proliferator-activated receptor alpha, PPARA) and lipid handling (perilipin 1, PLIN1; syntaxin-5, STX5; vesicle-associated membrane protein 4, VAMP4) (Fig. 5A).

In addition, INT-767 treatment reduced in the liver expression of pro-inflammatory genes (interleukin 6 (IL6); tumor necrosis factor alpha (TNFA)) and strongly induced anti-inflammatory/regulatory genes (forkhead box P3 (FOXP3); interleukin 10 (IL10)) (Fig. 5B).

\section{In vivo treatment with INT-767 enhances expression of genes involved in brown adipogenesis and mitochondrial biogenesis in preadipocytes}

Considering the effects of INT-767 on genes related to brown adipogenesis, mitochondriogenesis and insulin signaling, we examined spontaneous differentiation of rPADs isolated from the different experimental groups after 10 days of culture (Fig. 6).

Compared to rPADs from RD rabbits, HFD treatment significantly reduced mRNA expression of brown adipogenesis (bone morphogenetic protein 4, BMP4; homeobox protein Hox-9, HOXC9; LHX8; transmembrane protein 26, TMEM26), pro-fission proteins of mitochondria (mitochondrial fission 1 protein, FIS1) and cyclic-guanosine monophosphate (cGMP) signaling (soluble guanylyl cyclase beta-1, GCSB1) markers (Fig. 6A and B). In contrast, fatty acid binding protein 4 (FABP4), a white adipogenesis marker, was significantly increased $(P<0.05$; Fig. $6 \mathrm{~A})$. Treatment of HFD rabbits with INT-767 increased mRNA expression of several genes involved in brown adipogenesis (BMP4, CIDEA, HOXC9, LHX8, TMEM26), mitochondrial biogenesis (NRF1, TFAM), membrane respiratory chain (NADH dehydrogenase beta subcomplex 3, NDUFB3; NADH dehydrogenase beta subcomplex 5, NDUFB5; succinate dehydrogenase iron-sulfur subunit, $S D H B$; solute carrier family 25 member 12, SLC25A12), 


\begin{tabular}{|c|c|c|c|c|}
\hline $\begin{array}{l}\text { Journal of } \\
\text { Endocrinology }\end{array}$ & P Comeglio, I Cellai et al. & $\begin{array}{l}\text { INT-767 prevents metabolic } \\
\text { syndrome features }\end{array}$ & $238: 2$ & 115 \\
\hline
\end{tabular}

A
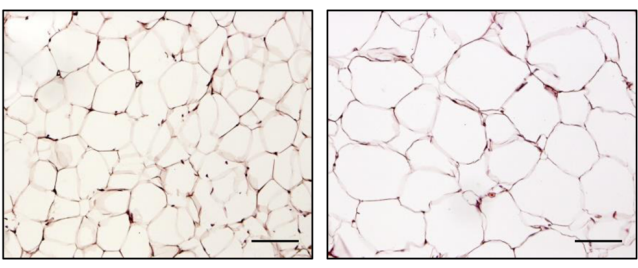

RD

HFD

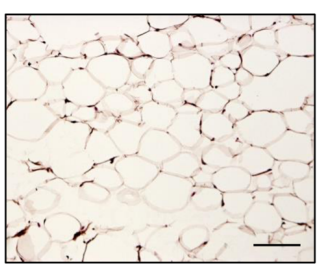

HFD+INT-767 $3 \mathrm{mg}$

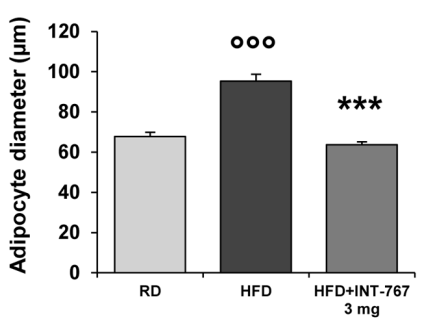

B
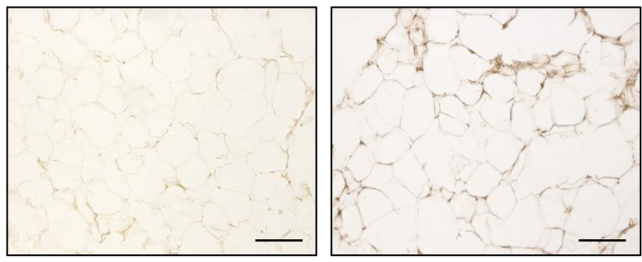

HFD

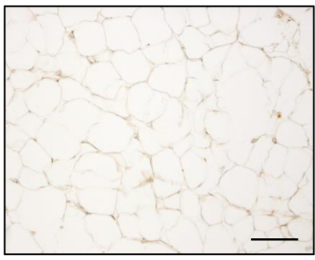

HFD+INT-767 $3 \mathrm{mg}$
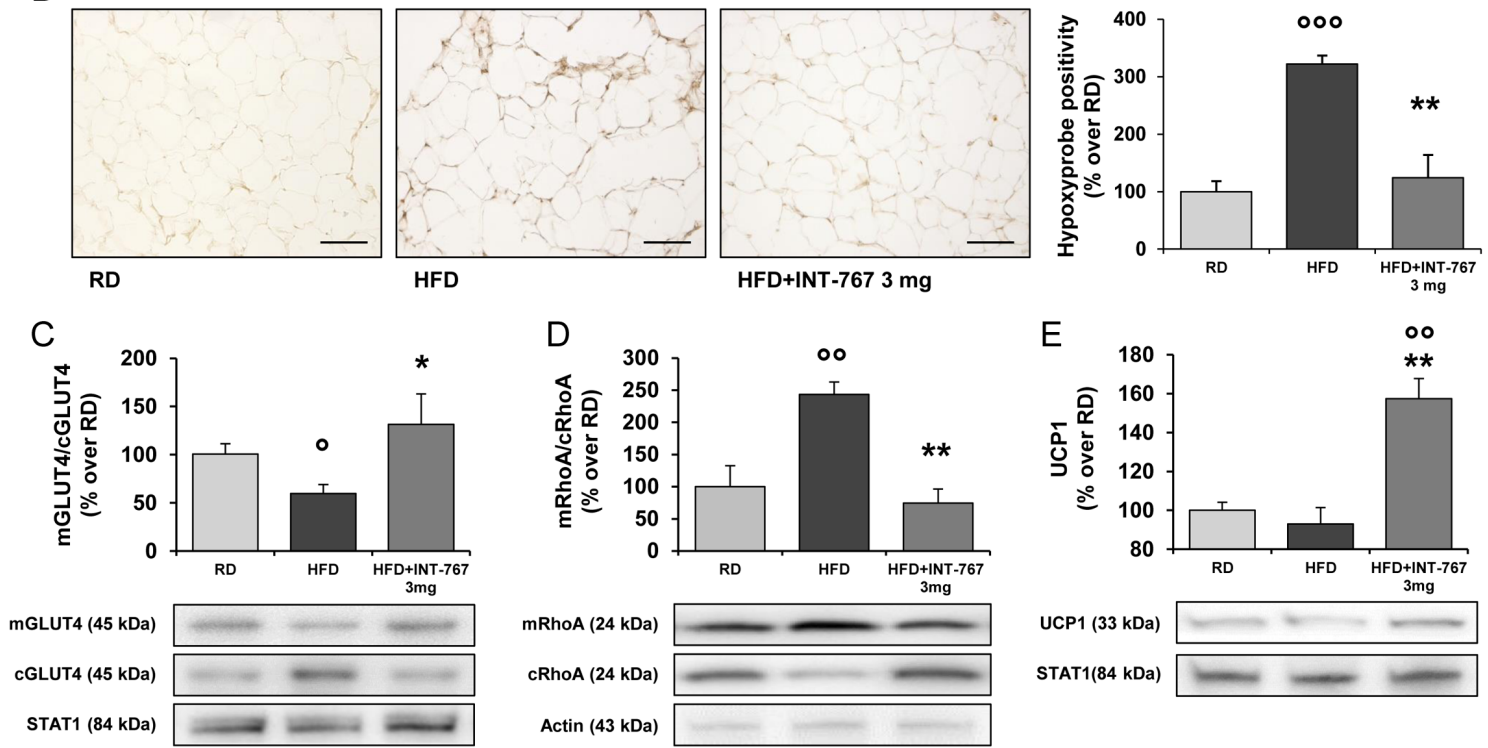

UCP1 (33 kDa)

STAT1 $(84 \mathrm{kDa})$

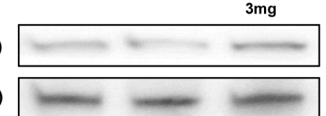

\section{Figure 4}

Effects of INT-767 treatment on adipocyte size, hypoxia, GLUT4 and RhoA membrane translocation and UCP1 in VAT sections from RD, HFD and HFD plus INT-767-treated rabbits. Panel A shows the histomorphometric analysis of adipocyte diameter $(\mu \mathrm{m})$ in the different experimental groups ( $n=3 / \mathrm{group}$ ) with hematoxylin-eosin staining (scale bar $=100 \mu \mathrm{m}$ ). Panel B shows the immunohistochemical staining of hypoxyprobe adducts in VAT sections. Hypoxyprobe adducts were revealed in hypoxic cells $\left(\mathrm{PO}_{2}<10 \mathrm{mmHg}\right)$ of VAT transverse sections by a hypoxyprobe-1-MAb (scale bar $\left.=100 \mu \mathrm{m}\right)$. RD optical density was taken as $100 \%$ in the computer-assisted quantitative image analysis of three independent experiments for each group. Panel $\mathrm{C}$ shows representative immunoblots with anti-GLUT4 and anti-signal transducer and activator of transcription 1 (STAT1) primary antibodies on membrane (m) and cytosolic (c) VAT protein extracts. The bar graphs show the optical density analyses of membrane/cytosol GLUT4 ratio ( $n=3 / g r o u p)$. Data are expressed as percentage of RD values. Panel $\mathrm{D}$ shows representative immunoblots with anti-RhoA and anti-actin primary antibodies on membrane ( $\mathrm{m}$ ) and cytosolic (c) VAT protein extracts. Bar graphs show the optical density analyses of membrane/cytosol RhoA ratio ( $n=3 /$ group). Data are expressed as percentage of RD values. Panel E shows representative immunoblots with anti-UCP1 and anti-STAT1 primary antibodies on VAT protein extracts from experimental rabbits. Bar graphs show the optical density analyses of UCP1 ( $n=3 /$ group). Data are expressed as percentage of RD values $\left({ }^{\circ} P<0.05\right.$, ${ }^{\circ 0} P<0.001,{ }^{\circ 00} P<0.0001$ vs RD; $* P<0.01, * * P<0.001, * * * P<0.0001$ vs HFD). A full colour version of this figure can be found at https://doi.org/10.1530/ JOE-17-0557.

pro-fission proteins of mitochondria (FIS1) and cGMP signaling (soluble guanylyl cyclase alpha-1, GCSA1; GCSB1, PKG1) (Fig. 6A and B). In contrast, compared to HFD, white adipogenesis markers (FABP4; peroxisome proliferator-activated receptor gamma, PPARG) were significantly reduced by in vivo dosing of INT-767 (Fig. 6A). Genes related to TGR5 signaling (PKA, EPAC1, CCND1, cAMP-responsive element modulator (CREM), CREB1, DIO2) were significantly increased by INT-767 treatment, whereas the FXR-dependent SHP expression was not affected (Fig. 6C).

\section{In vivo treatment with INT-767 enhances mitochondrial function and preserves their ultrastructure in preadipocytes}

To address the mechanisms underlying INT-767-induced VAT remodeling, we studied its effects on mitochondrial function in rPADs by using MitoTracker, a mitochondriatargeted fluorescent probe, which accumulates within active mitochondria (Fig. 7). Cells with similar shapes were chosen and time-lapse microscopic imaging was used to assess mitochondrial dynamics (fission-fusion events) 


\begin{tabular}{|c|c|c|}
\hline $\begin{array}{l}\text { Journal of } \\
\text { Endocrinologv }\end{array}$ & P Comeglio, I Cellai et al. & $\begin{array}{l}\text { INT-767 prevents metabolic } \\
\text { syndrome features }\end{array}$ \\
\hline
\end{tabular}
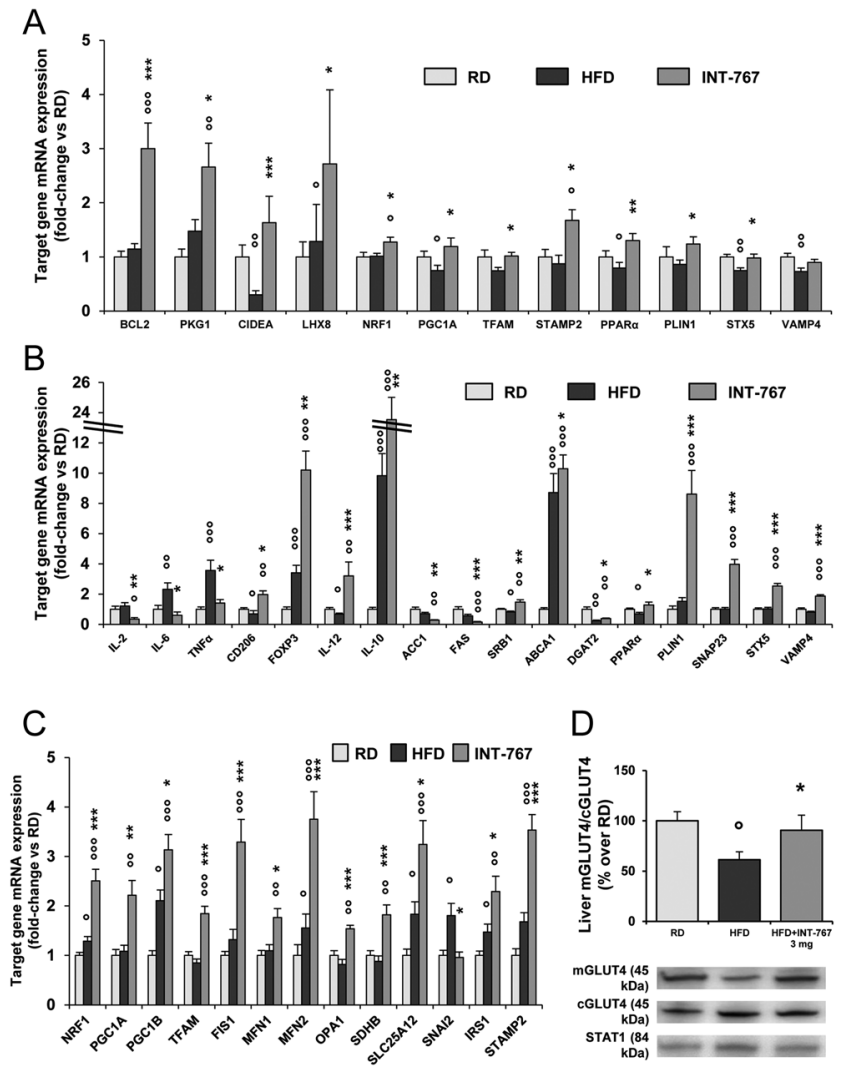

Figure 5

INT-767 treatment modulates mRNA expression of target genes in rabbit visceral fat (A) and liver (B and C) tissues. Data are calculated per the $2^{-\Delta \Delta C t}$ comparative method, using the $18 \mathrm{~S}$ ribosomal RNA subunit as reference gene for normalization and are reported as fold-change of mRNA expression over RD. Statistical analysis was performed using Kruskal-Wallis and Mann-Whitney tests $\left({ }^{\circ} P<0.05,{ }^{\circ} P<0.01,{ }^{\circ 0} P<0.001\right.$ vs $\mathrm{RD} ;{ }^{*} P<0.05, * * P<0.01, * * * P<0.001$ vs HFD). Panel D shows representative immunoblots with anti-GLUT4 and anti-STAT1 primary antibodies on membrane $(\mathrm{m})$ and cytosolic $(\mathrm{c})$ liver protein extracts. The bar graphs show the optical density analyses of membrane/cytosol GLUT4 ratio ( $n=3 /$ group). Data are expressed as percentage of RD values $\left({ }^{\circ} P<0.01\right.$ vs $\mathrm{RD} ;{ }^{*} P<0.05$ vs HFD).

(Fig. 7A and B). Computer-assisted measurement of mitochondria length in rPAD is reported in Fig. 7C. Mitochondria in RD rPADs showed heterogeneity in shape and length, resulting in a wide length distribution and an average length of $4.2 \mu \mathrm{m}$ (1.08-10.6 $\mathrm{m}$ ) (Fig. 7C). These mitochondria were particularly dynamic, continuously moving and changing shape (Fig. 7A, RD sequential images in Fig. 7B and Supplementary Movie 1). Conversely, in $\mathrm{PPAD}$ from HFD, mitochondria appeared randomly dispersed, aggregated, fragmented and nearly immobile (Fig. 7A), with less elongated mitochondrial network and an average length of $2.6 \mu \mathrm{m}(0.9-7.8 \mu \mathrm{m} ; P<0.0001$ vs RD) (Fig. 7C, HFD images in Fig. 7B and Supplementary Movie 2). Interestingly, the mitochondrial networks of rPADs from INT-767 group were highly dynamic (Fig. 7A),
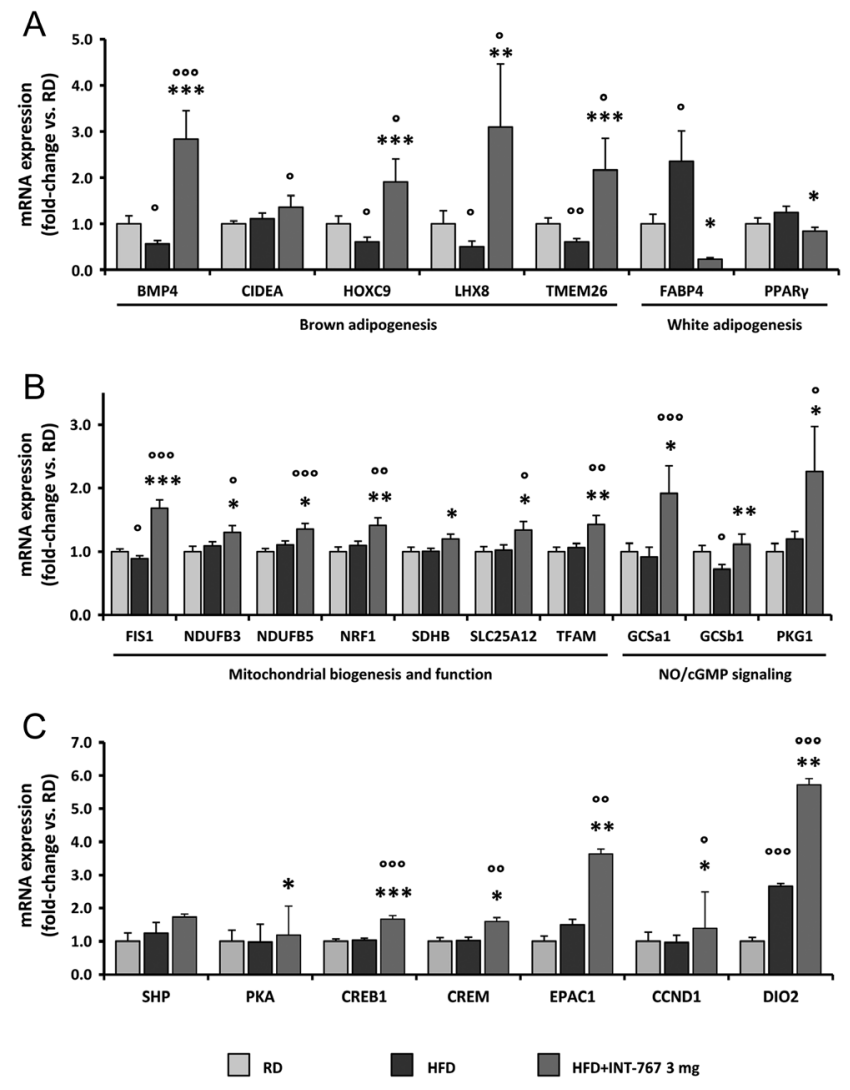

Figure 6

In vivo treatment with INT-767 enhances expression of genes involved in brown adipogenesis and mitochondrial biogenesis in preadipocytes. rPADs were analyzed after 10 days of spontaneous differentiation. Panel A reports the mRNA expression of genes involved in brown and white adipogenesis. Panel $B$ shows the mRNA expression of genes involved in mitochondrial biogenesis and function and involved in NO/cGMP signaling. Panel $\mathrm{C}$ displays the mRNA expression of FXR- and TGRdependent genes. Data are calculated per the $2^{-\Delta \Delta C t}$ comparative method, using the $18 \mathrm{~S}$ ribosomal RNA subunit as reference gene for normalization and are expressed as fold-change over RD cells (mean \pm S.E.M.) from at least three independent experiments. Statistical analysis was performed using the Mann-Whitney test $\left({ }^{\circ} P<0.05,{ }^{\circ} P<0.01,{ }^{\circ 0} P<0.001\right.$ vs RD; $* P<0.05, * * P<0.01, * * * P<0.001$ vs HFD).

continuously undergoing mitochondrial fission and fusion, with rapid changing in shape and length, with an average length of 3.6 $\mu \mathrm{m}(0.9-11.2 \mu \mathrm{m} ; P<0.0001$ vs HFD) (Fig. 7C, INT-767 images in Fig. 7B and Supplementary Movie 3).

We next studied changes in mitochondrial ultrastructure by transmission electron microscopy. The analysis further highlighted mitochondrial dysfunction in rPAD from HFD rabbits, with pronounced reduction of the cristae, up to complete loss, and increased electron density of the matrix $(P<0.0001$ vs RD; Fig. 7D, E, F and G). INT-767 treatment normalized HFD-induced reduction of mitochondrial cristae $(P<0.0001$ vs $H F D$; Fig. 7G), which resulted even higher than in $\mathrm{RD}$ rabbits $(P<0.0001$; Fig. 7G). 
A

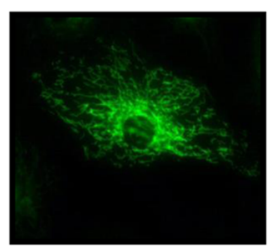

RD

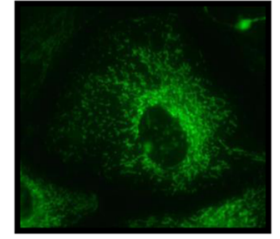

HFD

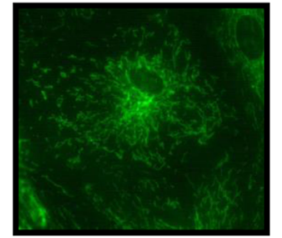

HFD+INT-767 $3 \mathrm{mg}$
B
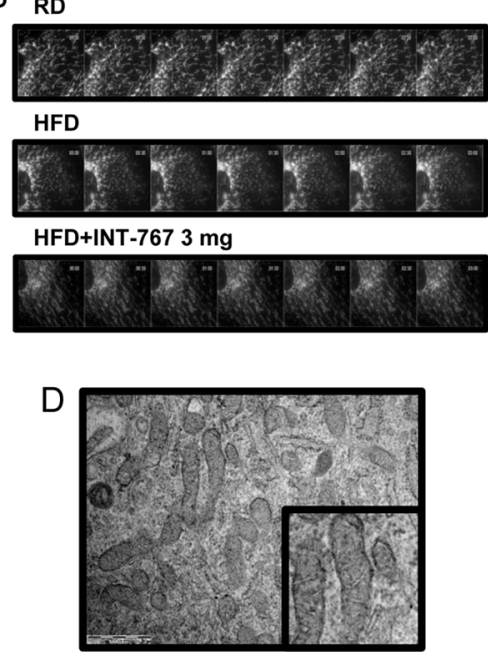

RD

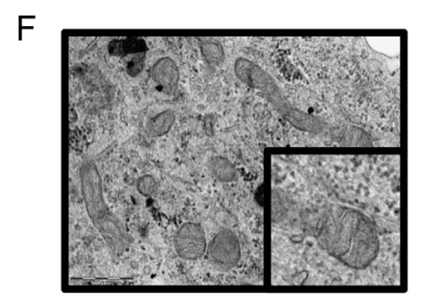

HFD+INT-767 $3 \mathrm{mg}$
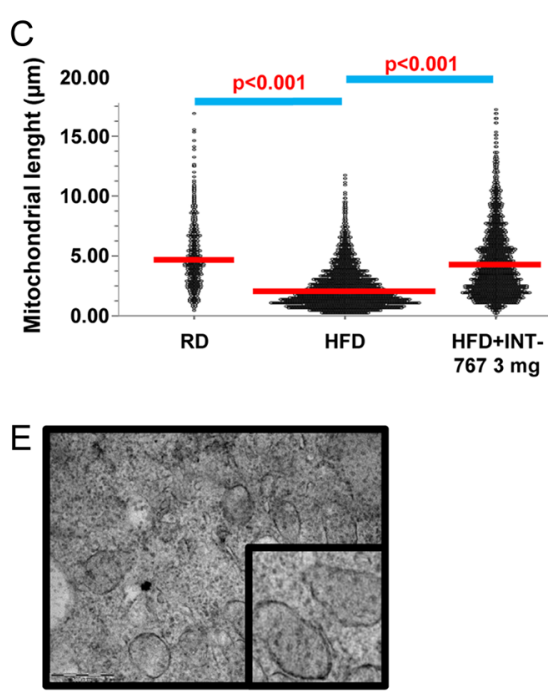

HFD

G

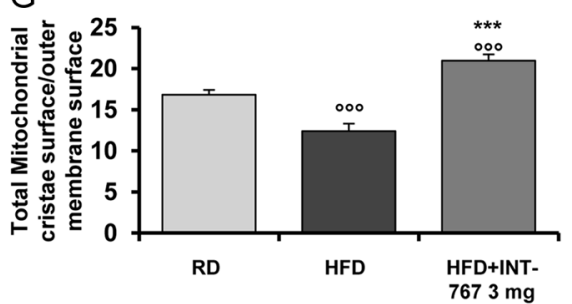

\section{Figure 7}

INT-767 treatment improves mitochondrial function. Panel A shows the mitochondrial function, visualized by incubation of the mitochondria-targeted fluorescent probe (MitoTracker) in rPAD isolated from RD, HFD and INT-767-treated rabbits. Panel B shows

representative time-lapse images of rPAD stained with MitoTracker and imaged for $3 \mathrm{~min}$. Computer-assisted measurement of mitochondria length is reported in panel C. For morphometric analysis of mitochondrial length, well-resolved mitochondria in the cell periphery were analyzed by ImageJ software. At least 50 individual mitochondrial structures in at least ten cells/ group were measured to determine mitochondrial length $(\mu \mathrm{m})$ distribution. Data were obtained from three independent experiments. Lines within the distribution represent the median values. Respective $P$ values are reported within the panel. INT-767 treatment normalizes mitochondrial ultrastructure in rPADs. rPADs were isolated from RD (panel D), HFD (panel E) and HFD + INT-767-treated (panel F) rabbits and analyzed by transmission electron microscopy (TEM). Bar graphs in panel G show the ratio of cristae membrane surface area to the outer membrane surface area, evaluated by iTEM image analysis software. Data are reported as mean \pm S.E.M. $\left({ }^{\circ 00} P<0.001\right.$ vs RD; $* * * P<0.001$ vs HFD). A full colour version of this figure can be found at https://doi.org/10.1530/JOE-17-0557.
Time-dependent accumulation of DHE-derived fluorescence served as a surrogate marker of spontaneous superoxide production in rPADs (Fig. 8A and B). As a function of time, RD rPADs did not show any DHEderived fluorescence accumulation (RD sequential 30-s images in Fig. 8A and Supplementary Movie 4). A time-dependent increase in superoxide generation was observed in rPAD from HFD (HFD sequential 30-s images in Fig. 8A and Supplementary Movie 5). INT-767 treatment significantly reduced superoxide production, compared to HFD (INT-767 sequential 30-s images in Fig. 8A and Supplementary Movie 6). Quantification of fluorescence intensity changes over time indicates that in vivo INT-767 treatment improved rPADs ability to reduce superoxide accumulation (Fig. 8B).

In order to assess the mitochondrial activity, we next evaluated oxygen consumption and quantification of intracellular ATP levels as readouts of mitochondrial function. In vivo INT-767 treatment significantly increased oxygen consumption, compared to RD and HFD rabbits groups (both $P<0.001$; Fig. $8 \mathrm{C}$ ), whereas no statistically significant difference was observed between RD and HFD experimental groups. The results of the intracellular ATP quantification in rPADs isolated from each experimental group are also reported. Compared to rPADs from RD and HFD rabbits, in vivo INT-767 treatment significantly decreased ATP intracellular levels (both $P<0.001$; Fig. 8D), whereas no difference was observed between RD and HFD experimental groups.

\section{INT-767 enhances adipogenic differentiation, lipid droplet formation and insulin sensitivity in DIM-induced preadipocytes}

As previously demonstrated (Maneschi et al. 2013), we here confirmed (Fig. 9A and C) that rPADs isolated from 
$A_{\text {RD }}$

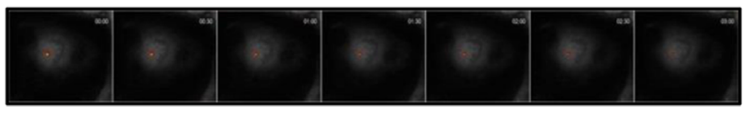

HFD

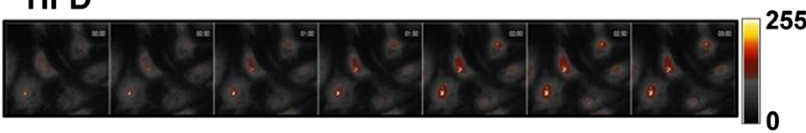

HFD+INT-767 $3 \mathrm{mg}$

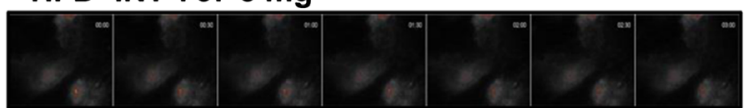

$B$.
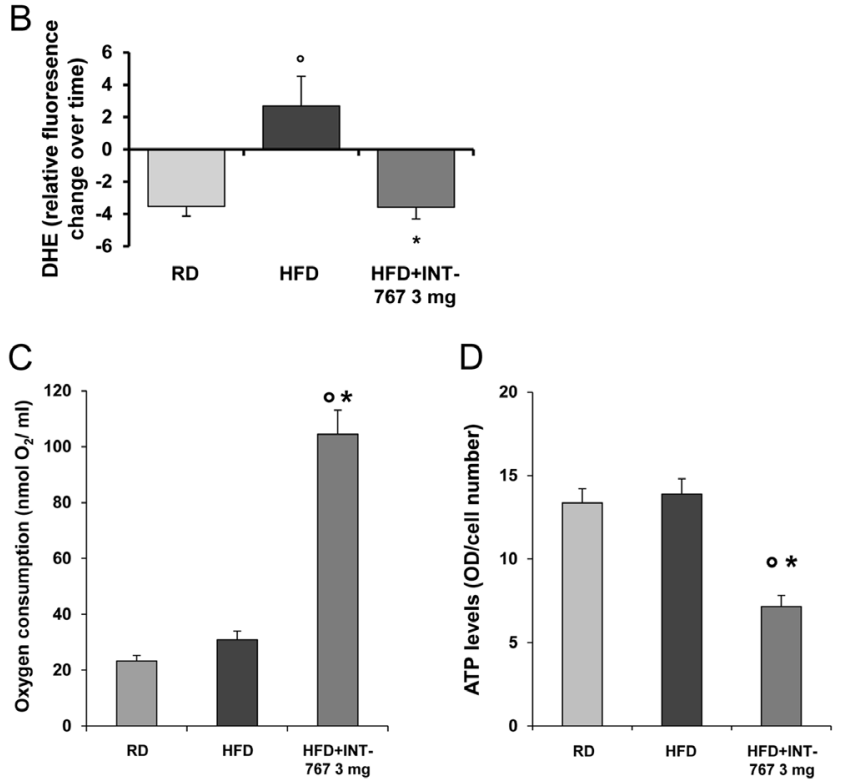

Figure 8

INT-767 treatment normalizes superoxide production in rPADs. Panel A displays representative time-lapse images of rPADs isolated from RD, HFD and INT-767-treated rabbits, stained with $10 \mu \mathrm{M}$ dihydroethidium (DHE) and imaged for $3 \mathrm{~min}$. Bar graphs show changes in integrated fluorescence intensity measured in the nuclei of rPAD cells during time-lapse imaging (B). Integrated intensity was measured in the initial $\left(\mathrm{I}_{\mathrm{o}^{\prime}}\right)$ and final $\left(\mathrm{I}_{180}\right)$ images of the time-lapse series. The relative change in fluorescence intensity over time in each cell was calculated as follows: $\left(\mathrm{I}_{180^{\prime}}-\mathrm{I}_{\mathrm{o}^{\prime}}\right) \times 100 / \mathrm{I}_{\mathrm{O}^{\prime}}\left({ }^{\circ} \mathrm{P}<0.001\right.$ vs RD $)$. Effects of INT-767 treatment on $\mathrm{O}_{2}$ consumption and intracellular ATP content in rPADs. Panel $C$ shows the oxygen consumption in rPADs isolated from RD, HFD and INT-767-treated rabbit after 10 days of spontaneous differentiation. It was measured by the Oxygraph system instrument. Bar graph shows the ratio of oxygen consumption normalized per $\mathrm{mL}$ of cell volume. Data are reported as the mean \pm S.E.M. of at least three independent experiments $\left({ }^{\circ} P<0.005\right.$ vs RD; ${ }^{*} P<0.005$ vs HFD). The quantification of intracellular ATP levels measured in rPADs isolated from each experimental group after spontaneous differentiation is shown in panel $D$. Luminescence was normalized to the number of total cells. Data are reported in RLU per cell as the mean \pm S.E.M. of at least three independent experiments $\left({ }^{\circ} P<0.005\right.$ vs $R D$; $\star P<0.005$ vs HFD). A full colour version of this figure can be found at https://doi.org/10.1530/JOE-17-0557. http://joe.endocrinology-journals.org https://doi.org/10.1530/JOE-17-0557 (c) 2018 Society for Endocrinology Published by Bioscientifica Ltd. Printed in Great Britain
HFD showed a reduced percentage of AdipoRed-positivity after DIM exposure, when compared to RD. Interestingly, in vivo INT-767 treatment induced a significant increase of AdipoRed-positive cells as compared with not only those isolated from HFD, but also from RD (Fig. 9A and C).

The intracellular lipid droplet content was analyzed by confocal microscopy in DIM-treated rPADs isolated from the different experimental groups (Fig. 9B). Lipid droplets of HFD rPADs were increased in volume compared to $\mathrm{RD}(P<0.001 ;$ Fig. 9D). In vivo INT-767 treatment normalized lipid droplet remodeling, reducing their volume $(P<0.0001$; Fig. 9D) and increasing droplets number $(P<0.05$; Fig. 9E), compared to rPADs from HFD rabbits.

In vivo INT-767 treatment also enhanced insulininduced uptake of ${ }^{3} \mathrm{H}-2$-deoxy-D-glucose (Fig. 9F). In DIM-induced rPADs from all experimental groups, insulin increased dose-dependently ${ }^{3} \mathrm{H}$-2-deoxy-D-glucose uptake, with similar $\mathrm{EC}_{50}$ values (shared $\mathrm{EC}_{50}=1.6 \pm 0.6 \mathrm{nM}$ ), but different maximal effect (Fig. 9F). In rPAD from HFD rabbits, insulin $E_{\max }$ was significantly reduced compared to $\mathrm{RD}$ rPADs $(\mathrm{HFD}=114.2 \pm 5.8 \%, \mathrm{RD}=180.1 \pm 9.5 \%, P=0.002$; Fig. 9F). In vivo treatment with INT-767 normalized the ability of rPADs to respond to increasing concentrations of insulin ( $E_{\max }:$ INT-767 $=174.9 \pm 9.1 \%, P=0.027$ vs HFD and $P=0.49$ vs RD; Fig. 9F). We also investigated the effect of in vitro treatment with INT-767 $(1 \mu \mathrm{M}$ for 10 days) on insulin-induced uptake of ${ }^{3} \mathrm{H}-2$-deoxy-Dglucose in DIM-induced rPADs isolated from HFD rabbits. Treatment in vitro with INT-767 significantly increased insulin $E_{\max }(176.1 \pm 27.5 \%)$, compared to untreated HFD (134.0 $\pm 12.7 \%, P=0.028$; Fig. 9G).

\section{INT-767 directly acts on differentiating rPAD via TGR5 stimulation}

To evaluate whether INT-767 effects in adipocytes were mediated by FXR or TGR5, we performed a series of in vitro experiments in rPADs. Increasing concentrations $(0.3-30 \mu \mathrm{M})$ of INT-767 dose-dependently stimulated cAMP production in rPAD from HFD (Fig. 10A). We next examined the effect of a 10-day stimulation with $1 \mu \mathrm{M}$ INT-767 in HFD rPADs, compared to an equimolar dose of INT-777 or OCA on genes related to FXR or TGR5 activation. By RT-PCR, we found that INT-767 significantly upregulated genes related to TGR5 signaling, including CCND1, CREB1, DIO2, EPAC1 and PKA, reaching levels similar to those observed with INT-777, (Fig. 10B). INT-767 increased TGR5 and SHP mRNA expression, even though the latter did not reach statistical significance 
A

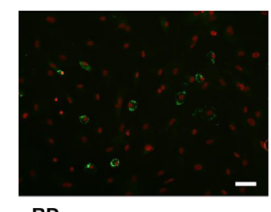

RD

B

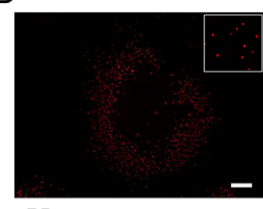

RD

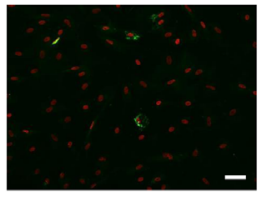

HFD

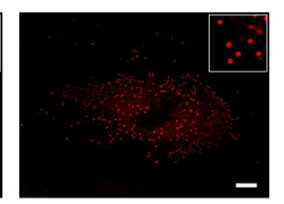

HFD

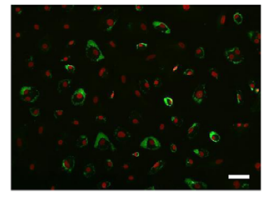

HFD+INT-767 3mg

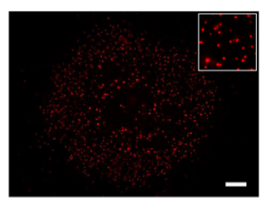

HFD+INT-767 $3 \mathrm{mg}$
C
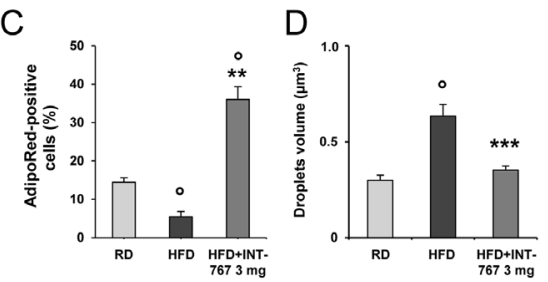

E

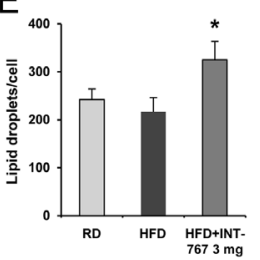

F
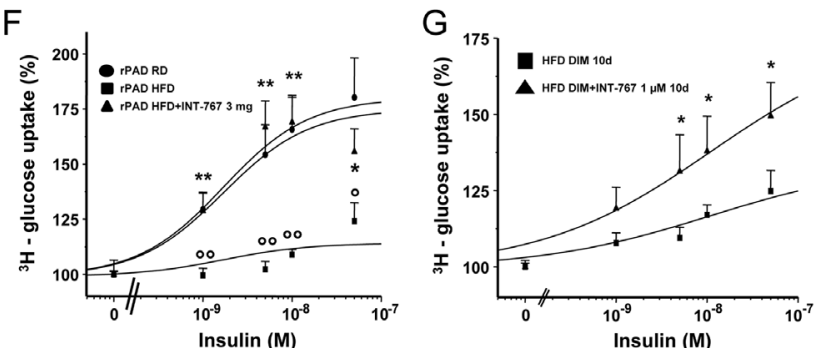

Figure 9

INT-767 treatment normalizes adipogenic differentiation, lipid droplet remodeling and insulin sensitivity in DIM-induced rPADs. RD, HFD and HFD+ INT-767 3 mg rPADs images were acquired with a Leica DM6000 microscope (magnification 10x) and were analyzed for adipogenic differentiation (panel A; scale bar $=10 \mu \mathrm{m}$ ). RD, HFD and HFD + INT-767 $3 \mathrm{mg}$ rPADs were imaged by confocal microscopy (magnification 63x) for the analysis of lipid droplets in AdipoRed-stained rPAD cells (panel B; scale bar $=10 \mu \mathrm{m}$ ). AdipoRed-positive cells were counted in at least four fields for each slide and expressed as percentage of total cells in three independent experiments (panel C). Confocal microscopy images were quantitatively analyzed using Volocity 5 software to measure volume ( $\mu \mathrm{m}^{3}$; panel D) and number (panel E) of lipid droplets within single cells. At least eight cells/group were analyzed. Results are reported as mean \pm S.E.M. ( ${ }^{\circ} P<0.001$ vs RD; $* P<0.05, * * P<0.001, * * * P<0.0001$ vs HFD). Panel F reports the effect of in vivo INT-767 treatment in DIM-induced rPADs on insulin-induced uptake of ${ }^{3} \mathrm{H}$-2-deoxy-D-glucose after exposure to increasing concentrations of insulin. Results are expressed in percentage over baseline (no insulin) and are reported as mean \pm S.E.M. of four different experiments, each performed in duplicate and using a different cell preparation per group. The relative $\mathrm{EC}_{50}$ and $E_{\max }$ values are reported in the text $\left({ }^{\circ} P<0.01,{ }^{\circ} P<0.001 \mathrm{HFD}\right.$ vs RD; ${ }^{*} P<0.05, * * P<0.001 \mathrm{HFD}+$ INT-767 $3 \mathrm{mg}$ vs HFD). Panel G shows the dose-response curves of radiolabeled ${ }^{3} \mathrm{H}$-glucose uptake in DIM-treated rPADs after exposure to increasing concentrations of insulin. Results are expressed as percentage over baseline and are reported as mean \pm s.E.M. of four different experiments, each performed in duplicate and using a different cell preparation per group. The relative $\mathrm{EC}_{50}$ and $E_{\max }$ values are reported in the text (* $P<0.05$ vs $\mathrm{HFD}$ ). A full colour version of this figure can be found at https://doi.org/10.1530/JOE-17-0557.

(Fig. 10B). OCA significantly increased mRNA expression of PKA and TGR5 (Fig. 10B). Several genes related to mitochondrial biogenesis and function (FIS1; mitofusin 1, MFN1; mitofusin 2, MFN2; NDUFB3; NRF1) and to brown differentiation ( $L H X 8)$ were increased by INT-767 (Fig. 10C). The large majority of these genes were also upregulated by INT-777, but not by OCA (Fig. 10C). Furthermore, both in vitro treatments with OCA and INT-767 significantly increased mRNA expression of GLUT4, STAMP2 and insulin receptor substrate 1 (IRS1) in rPAD from HFD rabbits, whereas INT-777 showed a milder effect on these insulin signaling markers (Fig. 10C). To further ascertain the role of TGR5 activation in mediating INT-767 effect on rPADs, INT-767 was tested in vitro with or without the cell-permeable protein kinase A inhibitor (PKI 14-22 amide, myristoylated, $1 \mu \mathrm{M}$ ) or forskolin $(10 \mu \mathrm{M})$. Co-treatment of INT-767 with PKI significantly blunted all the INT-767-induced effects on genes related to TGR5 signaling (CREB1; DIO2; PKA) and mitochondrial function (FIS1; MFN1; MFN2; NDUFB3; NRF1) (Fig. 10D). Forskolin, as a positive control, could mimic the effect of INT-767 on the TGR5 downstream effectors (Fig. 10D).
UCP1 expression in HFD rPADs (Fig. 10E, F, G and $\mathrm{H}$ ) was analyzed with fluorescent microscope immunocytochemistry after in vitro treatment with INT-767 $1 \mu \mathrm{M}$ or an equimolar dose of OCA or INT-777. INT-767 treatment was able to promote significant brown adipogenesis, expressed as percentage of UCP-1-positive cells over total cells, compared to untreated rPADs $(P<0.001$; Fig. 10I). Likewise, INT-777 in vitro treatment also determined a significant UCP1 increase, whereas the OCA-induced increase did not reach statistical significance (Fig. 10I). Accordingly, in vitro INT-767 treatment $(1 \mu \mathrm{M})$ determined a significant increase, when compared to untreated HFD rPADs of oxygen consumption (HFD: $30.83 \pm 3.15 \mathrm{nmol} \mathrm{O}_{2} / \mathrm{mL} ; \quad \mathrm{HFD}+\mathrm{INT}-7671 \mu \mathrm{M}$ : $\left.69.44 \pm 14.74 \mathrm{nmol} \mathrm{O}_{2} / \mathrm{mL} ; P<0.05\right)$, as well as a reduction of ATP formation (HFD: $13.89 \pm 0.92$ relative light unit (RLU)/cell number; HFD+INT-767 $1 \mu \mathrm{M}$ : $9.34 \pm 0.59$ RLU/cell number; $P<0.005)$.

\section{SiRNA in hPADs}

siRNA targeting of FXR and TGR5 was evaluated in hPADs isolated from VAT samples obtained from obese patients 
A
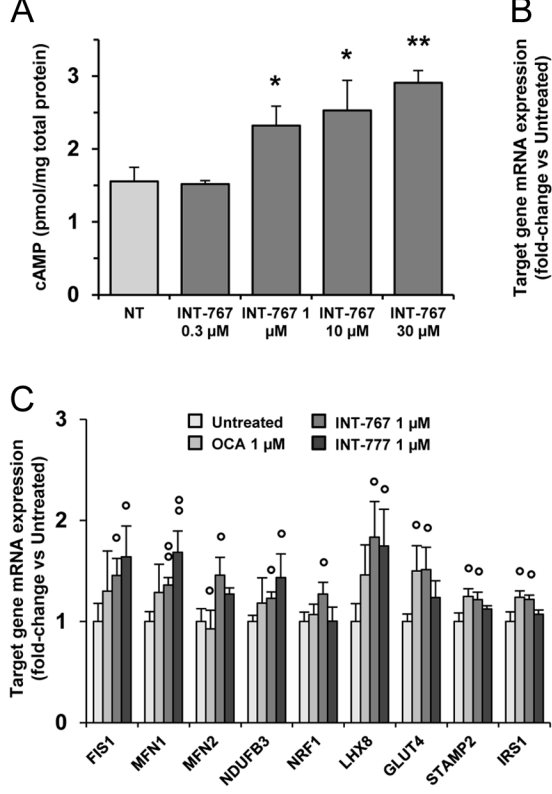

E

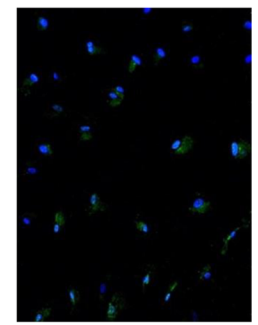

Untreated

$\mathrm{H}$

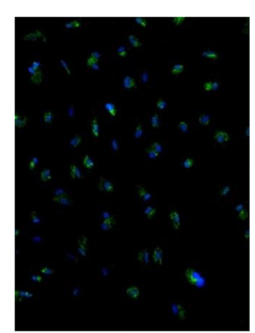

INT-777 $1 \mu \mathrm{M}$
$\mathrm{F}$

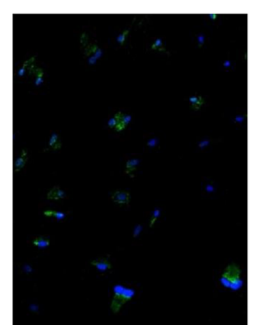

OCA $1 \mu \mathrm{M}$

I

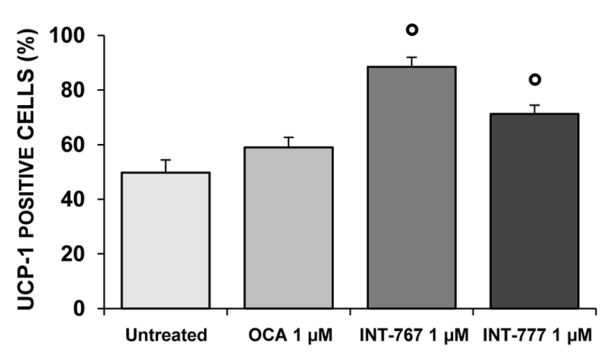

INT-767 $1 \mu \mathrm{M}$
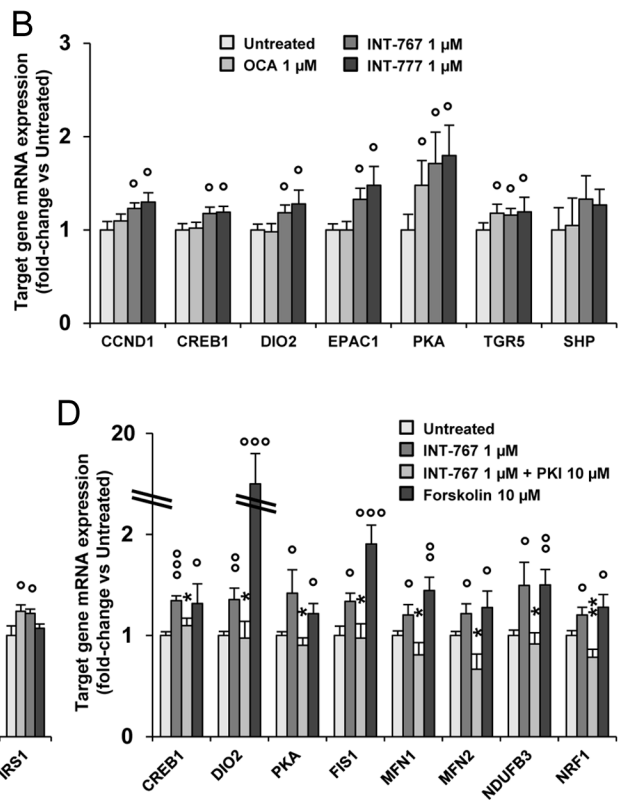

G

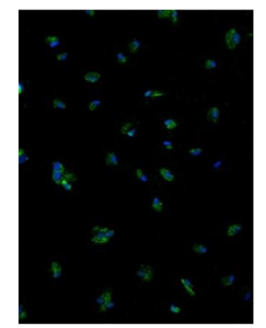

Figure 10

INT-767 directly acts on differentiating rPAD via TGR5 stimulation. Panel A shows the effect of the in vitro treatment with INT-767 on the intracellular CAMP production in rPADs cells isolated from the HFD experimental group. The basal CAMP concentration of untreated serum-starved cells was taken as control (NT). Data are expressed as pmol of cAMP per mg of total proteins and reported as mean \pm S.E.M. of three independent experiments $(* P<0.05$, $\star * P<0.01$ vs NT). Panel B reports the in vitro effects on TGR5-related genes mRNA expression by 10 -day stimulation with OCA $(1 \mu \mathrm{M})$, INT-767 $(1 \mu \mathrm{M})$ or INT-777 $(1 \mu \mathrm{M})$ of HFD rPADs. Data are calculated per the $2^{-\Delta \Delta \mathrm{Ct}}$ comparative method, using the $18 \mathrm{~S}$ ribosomal RNA subunit as reference gene for normalization and are reported as fold-change of mRNA expression over untreated cells. Panel $C$ shows the in vitro effects on mRNA expression of mitochondria-related genes after 10-day stimulation of HFD rPADs with OCA $(1 \mu \mathrm{M})$, INT-767 $(1 \mu \mathrm{M})$ or INT-777 $(1 \mu \mathrm{M})$. Panel D displays the in vitro effects on HFD rPADs following 18-h stimulation with INT-767 $(1 \mu \mathrm{M})$ in the presence or absence of the specific highaffinity PKA inhibitor PKI $(10 \mu \mathrm{M})$. Forskolin $(10 \mu \mathrm{M})$ was used as positive control $\left({ }^{\circ} P<0.05\right.$, ${ }^{\circ} P<0.01,{ }^{\circ \circ} P<0.001$ vs untreated; $* P<0.05$, $* * P<0.01$ vs INT-767 $1 \mu \mathrm{M})$. Panels E, F, G, H and I show the effect of OCA, INT-767 and INT-777 treatment for 10 days on UCP-1 expression in rPADs. Displayed are representative images of HFD rPADS, left untreated (E) and treated with OCA (F), INT-767 (G) and INT-777 (H). Panel I shows the percentage of UCP-1 positive cells calculated by counting the stained cells in ten fields per slide of three different experiments normalized on total cells (DAPI stained) $\left({ }^{\circ} P<0.001\right.$ vs untreated; scale bar $=50 \mu \mathrm{m})$. A full colour version of this figure can be found at https://doi. org/10.1530/JOE-17-0557.

undergoing bariatric surgery, which represent the human equivalent of the HFD rabbit. Positive controls (GAPDH), negative controls and target genes (FXR and TGR5) analyses showed that the experimental procedure was functional (Fig. 11A, B and C). As readouts for the specific receptors silencing, we selected markers for TGR5-dependent mitochondrial activity (FIS1) and brown adipogenesis (beta-3 adrenergic receptor, $A D R B 3$ ), and FXR-dependent insulin signaling (IRS1). Both FIS1 and ADRB3 mRNA expression, upregulated by INT-767 and INT-777 in vitro treatment, were significantly reduced by TGR5 silencing, whereas OCA treatment and FXR silencing did not appear to modulate these genes expression (Fig. 11D and E).
Similarly, IRS1 expression was upregulated by OCA and INT-767 in vitro treatment and significantly reduced by FXR silencing. Accordingly, IRS1 gene expression was neither induced nor downregulated by INT-777 treatment and TGR5 silencing, respectively (Fig. 11F).

\section{INT-767 treatment inhibits HFD-induced NASH}

As INT-767 treatment improves HFD-induced VAT alterations and ameliorates several features in dysfunctional adipocytes, including insulin resistance, we tested whether in vivo treatment with INT-767 could improve MetS-associated NASH, the hepatic hallmark of 
A
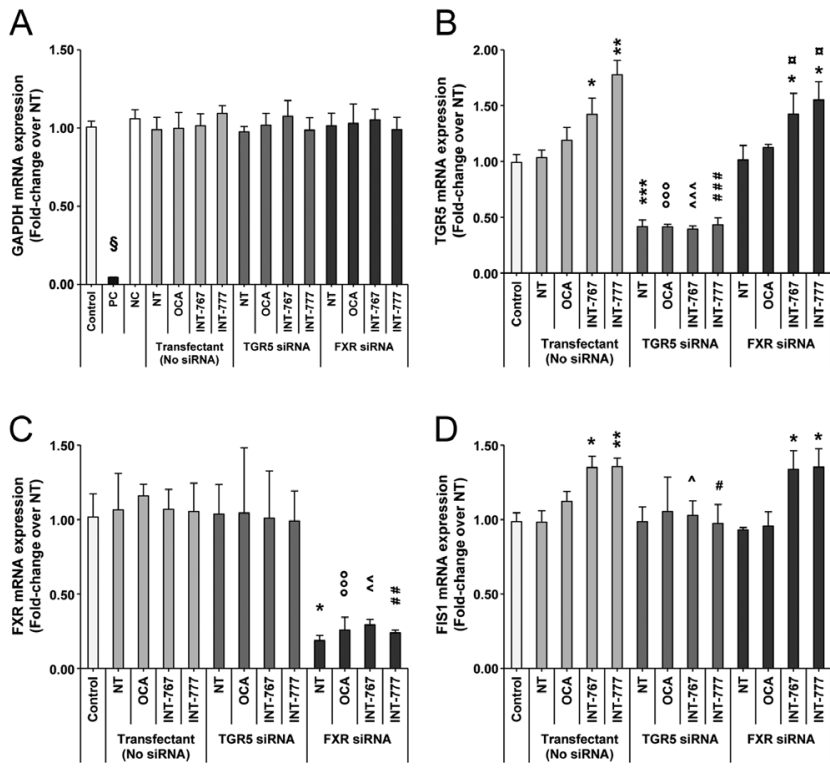

$\mathrm{E}$

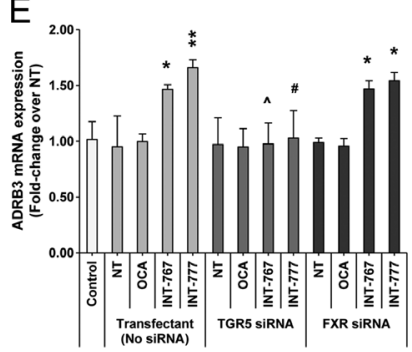

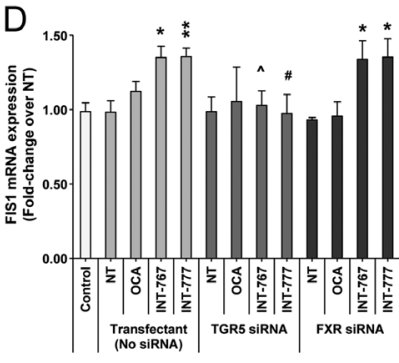

$\mathrm{F}$

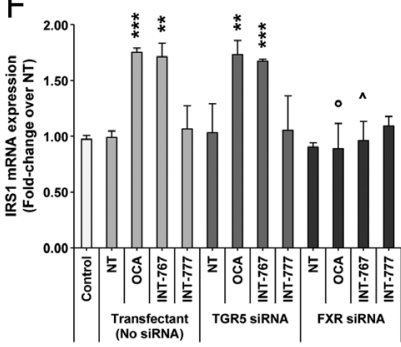

\section{Figure 11}

FXR and TGR5 silencing in hPADs. Panel A shows the relative mRNA expression of GAPDH in Control (untreated) cells, positive control (PC; GAPDH silencing), negative control (NC; non-targeting pool) and after incubation with transfectant without siRNA, TGR5 siRNA and FXR siRNA in untreated (NT) or treated (OCA, INT-767, INT-777) hPADs. Panels B and $C$ show the relative mRNA expression of TGR5 and FXR, respectively. Panels D, E and F show the relative mRNA expression of FIS1, ADRB3 and IRS1, respectively, in Control (untreated) cells and after incubation with transfectant without siRNA, TGR5 siRNA and FXR siRNA in untreated (NT) or treated (OCA, INT-767, INT-777) hPADs. Experiments were performed in triplicate using four different hPAD preparations. Data are calculated per the $2-\Delta \Delta C \mathrm{Ct}$ comparative method, using the $18 \mathrm{~S}$ ribosomal RNA subunit as reference gene for normalization. Results are expressed in fold-change over Control (untreated) cells and are reported as mean \pm S.E.M. on an arithmetic scale graph. $\$ P<0.001$ vs Control; $P<0.05$ vs NT (FXR siRNA); ${ }^{*} P<0.05, * * P<0.01, * * * P<0.001$ vs NT (transfectant); ${ }^{\circ} P<0.05,{ }^{\circ 0 \circ} P<0.001$ vs OCA (transfectant); ${ }^{\wedge} P<0.05,{ }^{\wedge} P<<0.01,{ }^{\wedge \wedge} P<0.001$ vs INT-767 (transfectant); $\# P<0.05, \# P<0.01, \# \# P<0.001$ vs INT-777 (transfectant).

insulin resistance. Feeding rabbits a HFD for 12 weeks induced severe histological alterations within the liver (Fig. 12), including hypoxia (A), fat accumulation (B), collagen deposition (C) and mononuclear cell infiltrates (D). In addition, HFD increased type 1 pro-inflammatory macrophages, as shown by increased staining with M1 marker IRF5 (Fig. 12E). All these alterations were significantly counteracted by INT-767 treatment (Fig. 12A,

B, C, D and E). HFD treatment also had marked effects on gene transcripts in liver homogenates (Fig. 5B), inducing a significant increase of pro-inflammatory genes (IL2; IL6; TNFA), which was paralleled by a decrease of cluster of differentiation 206 (CD206) expression, a marker of antiinflammatory M2 macrophages. INT-767 significantly reduced mRNA expression of all the above mentioned pro-inflammatory genes, while significantly increasing the anti-inflammatory ones, including FOXP3 and IL10 (Fig. 5B). Interestingly, INT-767 treatment was also able to markedly increase the circulating level of the antiinflammatory cytokine IL10 (RD: $16.07 \pm 2.26 \mathrm{pg} / \mathrm{mL}$; HFD: $12.88 \pm 1.60 \mathrm{pg} / \mathrm{mL} ; \quad$ HFD + INT-767: $32.50 \pm 6.01 \mathrm{pg} / \mathrm{mL}$, $P=0.007$ vs HFD). INT-767 also induced a significant reduction of hepatic expression of genes related to de novo fatty acid synthesis (acetyl-CoA carboxylase 1, ACC1; fatty acid synthase, $F A S$ ), while increased those related to lipid uptake (scavenger receptor B1, SRB1), efflux (ATP-binding cassette transporter 1, ABCA1), metabolism (diacylglycerol O-acyltransferase 2, DGAT2; PPARA) and lipid droplet formation (PLIN1; synaptosomal-associated protein 23, SNAP23; STX5; VAMP4) (Fig. 5B). Markers of mitochondrial biogenesis (NRF1; PGC1A; peroxisome proliferator-activated receptor gamma coactivator 1-beta, PGC1B; TFAM) and function (FIS1; MFN1; MFN2; dynamin-like protein, OPA1; SDHB; SLC25A12) were also significantly increased by INT-767. Finally, we found a significant reduction of the hepatocyte-derived profibrogenic agent SNAI2 in INT-767-treated rabbits as compared to HFD (Fig. 5C).

In the liver, INT-767 treatment stimulated the expression of genes related to insulin signaling, including IRS1 and STAMP2 (Fig. 5C). Western blot analysis showed that plasma membrane translocation of GLUT4, significantly hampered by HFD, was normalized by INT-767 treatment (Fig. 5D).

\section{Discussion}

The limited ability of adipocytes to store and metabolize excess nutrients is a key element in determining insulin resistance, leading to ectopic lipid deposition in tissues such as liver. Feeding rabbits HFD for 12 weeks was associated with a substantial deterioration of preadipocyte insulin sensitivity, which was coupled to multiple mitochondrial ultrastructural and functional alterations (Maneschi et al. 2016). Mitochondrial morphology and dynamics are hallmarks of mitochondrial functions (Liesa \& Shirihai 2013). In healthy, dynamic mitochondria, 
A

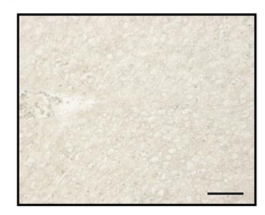

RD

B

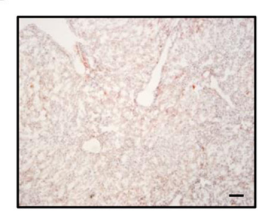

RD

C

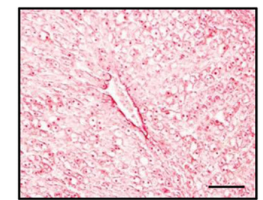

RD

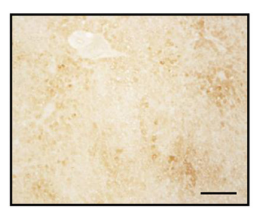

HFD

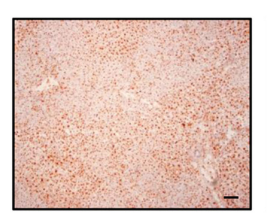

HFD

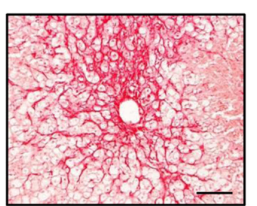

HFD

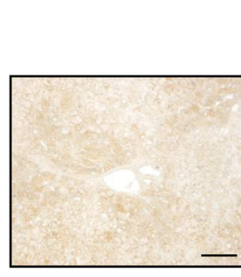

HFD+INT-767 3mg

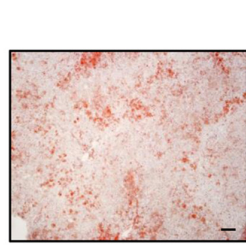

HFD+INT-767 3mg

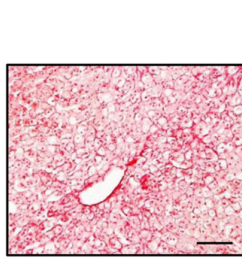

HFD+INT-767 3mg
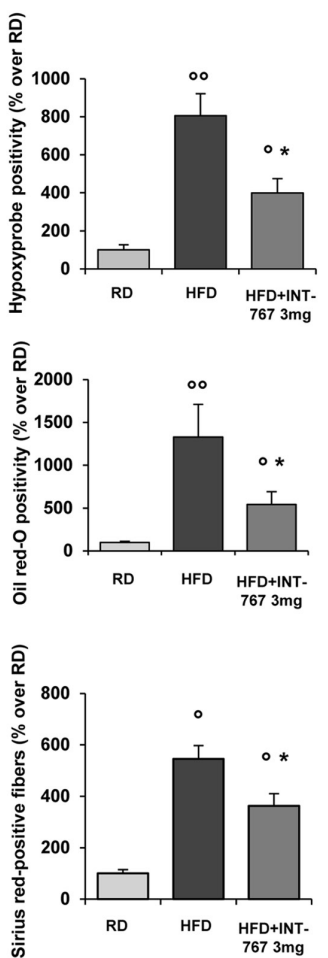

D

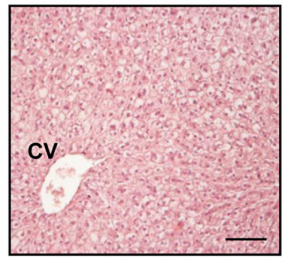

RD

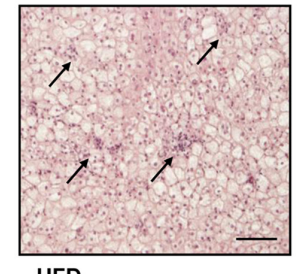

HFD

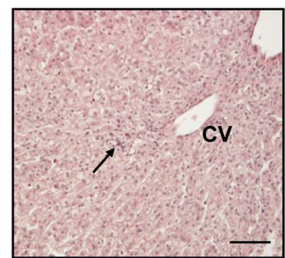

HFD+INT-767 3mg
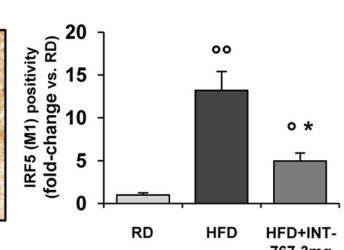

Figure 12

INT-767 treatment inhibits NASH development. Panel A shows the immunohistochemical staining of hypoxyprobe adducts in liver sections from RD, HFD and HFD + INT-767 (3 mg/kg) rabbits. RD optical density was taken as $100 \%$ in the computer-assisted quantitative image analysis of three independent experiments/group $\left({ }^{\circ} P<0.001\right.$, ${ }^{\circ} P<0.0001$ vs RD; ${ }^{*} P<0.001$ vs HFD). Panel B displays lipid accumulation in liver sections, as revealed by Oil Red-O staining. Data are reported as percentage over $\operatorname{RD}\left({ }^{\circ} P<0.05,{ }^{\circ} P<0.01\right.$ vs RD; ${ }^{*} P<0.05$ vs HFD). Panel $C$ reports Sirius Red staining of liver sections. In each section, Picrosirius red-positive areas were measured in five non-overlapping randomly chosen fields and were determined by excluding staining artifacts. Data are expressed as percentage over RD $\left({ }^{\circ} P<0.0001\right.$ vs $\mathrm{RD} ;{ }^{*} P<0.05$ vs HFD). Panel D shows representative images from hematoxylin/eosin staining of liver sections. Arrows indicate mononuclear cell infiltrates (CV, centrolobular vein). Panel E displays the immunohistochemical localization of IRF5 (M1 macrophage marker), whose positivity is quantified and expressed as fold-change over $\mathrm{RD}\left({ }^{\circ} P<0.01,{ }^{\circ} \mathrm{P}<0.001\right.$ vs $\mathrm{RD}$; $* P<0.01$ vs HFD). All images are representative of those obtained in at least three rabbits/group (scale bar $=100 \mu \mathrm{M}$ ). A full colour version of this figure can be found at https://doi.org/10.1530/ JOE-17-0557. the inner membrane is characterized by abundant cristae that provide a higher surface area, allowing for a greater buffering of reactive oxygen species, in concert with the endoplasmic reticulum (Newmeyer \& Ferguson-Miller 2003). Accordingly, mitochondria in rPADs from HFD rabbits were small, immobile and highly fragmented, with loss of mitochondrial cristae and increased superoxide formation. In vivo INT-767 treatment improved these mitochondrial alterations in rPADs, coupled with a reduced superoxide content and ATP synthesis and increased oxygen consumption. These mitochondrial functional improvements were associated with increased UCP1 expression induced by INT-767.

http://joe.endocrinology-journals.org https://doi.org/10.1530/JOE-17-0557 (c) 2018 Society for Endocrinology Published by Bioscientifica Ltd. Printed in Great Britain
Lipid droplet remodeling was also markedly improved by in vivo INT-767 treatment. Lipid droplets closely interact with the endoplasmic reticulum and the mitochondria (Aon et al. 2014), and shield other cell organelles from the potential lipotoxicity of fatty acids (Nguyen et al. 2017), representing an efficient way to maintain ROS within levels compatible with signaling while ensuring energy supply as well as insulin sensitivity (Kelley et al. 2002, Bach et al. 2003, Toledo et al. 2006, Gao et al. 2010, Mason \& Watt 2015). In line with this view, in vivo exposure to INT-767 not only improved mitochondrial function and lipid droplets handling but also normalized insulin sensitivity in preadipocytes. 
A striking finding of this study is that in rPADs from INT-767-treated rabbits, the expression of genes related to brown adipogenesis and cGMP signaling, a pathway playing a pivotal role in adipocyte remodeling toward a brown phenotype (Haas et al. 2009, Mitschke et al. 2013, Hoffmann et al. 2015, Maneschi et al. 2016), was significantly upregulated, compared to rPADs from HFD animals. This was accompanied by a marked reduction of white adipogenesis-related markers and an increase of mitochondrial biogenesis and function markers. These results indicate an overall improved metabolism and/or a direct effect on adipocytes of in vivo INT-767 treatment.

Interestingly, the gut-restriced FXR agonist fexaramine (Fex) induces enteric FGF15 in mice with diet-induced obesity, without activating FXR target genes in the liver (Fang et al. 2015). Fex promotes metabolic improvements, with reduction of diet-induced weight gain, bodywide inflammation and hepatic glucose production, while enhancing thermogenesis and browning of white adipose tissue (Fang et al. 2015). These results suggest that gut-restricted FXR activation also might contribute to the overall improvement of metabolic alterations. Interestingly, we found that both FXR and TGR5 were highly expressed in intestine.

Therefore, in order to try to elucidate whether INT-767 could directly act on adipose tissue, we performed a series of in vitro experiments. Exposure of rPADs from HFD animals to INT-767 for 10 days induced UCP1 expression and oxygen consumption, which were associated with reduction of ATP formation. The UCP1-induced higher respiration and the uncoupling of the oxidative phosphorylation process results in dissipation of oxidation energy as heat (Rousset et al. 2004). These observations are well in line with the currently accepted model wherein regulation of UCP1 coupled with a reduction of ATP serves as a central point of brown adipogenesis (Klingenspor et al. 2017, Fromme et al. 2018). Accordingly, in INT-767-treated cells, a substantial increase in the mRNA expression of NRF1 was also observed. NRF1 is considered as a major player for UCP1 expression and BAT thermogenic function (Bartelt et al. 2018). INT-767 also induced genes related to mitochondrial biogenesis and function. In these experiments, INT-767 behaves mainly as a TGR5 agonist, directly activating the cAMP/PKA pathway. These effects of INT-767 were mimicked by an equimolar concentration of the selective TGR5 agonist, INT-777, and by the direct PKA activator, forskolin, while abrogated by the selective PKA inhibitor, PKI. This conclusion is also supported by the finding that INT-767 induced dose-dependently cAMP production and, moreover, both in vitro and in vivo INT-767 treatments induced in rPADs the expression of TGR5-dependent genes. Our findings are consistent with the recent observation that another dual FXR/TGR5 agonist could promote in vitro trans-differentiation of murine 3T3-L1 preadipocytes into a beige phenotype by increasing intracellular cAMP (Carino et al. 2017). TGR5 is generally implicated in the induction of energy expenditure in BAT, reducing in mice diet-induced obesity and insulin resistance (Watanabe et al. 2006). Treatment of human brown adipocytes with BAs or TGR5 agonists (INT-777) significantly enhances energy expenditure and mitochondrial respiration through an UCP1-dependent mechanism (Broeders et al. 2015).

The observed changes in mitochondria and lipid droplets features, along with the modulation of the gene expression profile, indicate that INT-767 could reshape VAT by promoting, mostly through TGR5 activation, a differentiation program of preadipocytes toward a brown phenotype, leading to metabolically healthier mitochondria. Accordingly, we detected an induction of UCP1 in VAT homogenates derived from in vivo INT-767-treated rabbits, compared to untreated HFD. Several genes related to mitochondriogenesis, proliferation and browning were also increased in VAT homogenates from INT-767-treated rabbits, compared to HFD. These effects were accompanied by increased mRNA expression of lipid droplet genes and PPARA, a potent regulator of differentiation, fatty acid oxidation and insulin-stimulated glucose uptake (Goto et al. 2011). In VAT, INT-767 treatment also normalized insulin-regulated membrane translocation of GLUT4 (Govers 2014) and RhoA (Kanda et al. 2006) and increased expression of STAMP2 (Wellen et al. 2007). Accordingly, in vitro treatment with INT-767 in rPADs significantly upregulated the expression of GLUT4, STAMP2 and IRS1. These in vitro effects, mimicked by OCA but not by INT-777, indicate that FXR activation mostly contributed to improved insulin signaling by INT-767.

The use of rabbits as experimental models presents some limitations. Specifically, this model is unsuitable for knockdown studies, as gene disruptions are difficult to achieve in rabbits (Kawano \& Honda 2017). In particular, in vitro silencing of FXR/TGR5 in rabbit PADs is not possible with the currently available commercial tools for silencing. One possible approach could be represented by the use of the CRISPR/Cas9 technology, in order to further clarify the specific contribution of each receptor. Meanwhile, we decided to carry out siRNA silencing experiments on human PADs isolated from obese subjects undergoing bariatric surgery, which represent the human equivalent of the HFD rabbit. We demonstrated that FXR 
silencing downregulated IRS1 mRNA expression, readout of FXR-induced insulin sensitivity. Likewise, siRNA directed toward TGR5 caused a marked reduction in FIS1 and ADRB3 mRNA expression, respectively markers of mitochondrial biogenesis and brown adipogenesis.

In vivo INT-767 dosing in HFD rabbits also prevented other features related to insulin insensitivity, such as adipocytes hypertrophy and hypoxia. We found that FXR was highly expressed not only in adipocytes but also in endothelial cells of the VAT vascular network. Increasing evidence supports a role for FXR in the process of vascular remodeling, in part through an effect on endothelial cells (He et al. 2006, Li et al. 2009, Hageman et al. 2010, Miyazaki-Anzai et al. 2010, Vignozzi et al. 2017). Therefore, a FXR-dependent activity of INT-767 on a cellular target different from adipocytes (i.e. endothelial cells) in VAT should be further investigated.

Overall, our results suggest that INT-767 exerts two distinct actions in VAT, promoting a TGR5-mediated browning program and a FXR-mediated improved insulin signaling. Nevertheless, it should be noticed that in vitro treatment with OCA in rPADs was also able to induce the expression of TGR5 and its downstream effector PKA. Similarly, in vivo administration of OCA upregulated not only the expression of SHP but also of the expression of TGR5 in VAT. Accordingly, a recent study identifies TGR5 as a FXR target gene due to the putative FXR-responsive element localized in the TGR5 promoter (Pathak et al. 2017), thus suggesting an intimate cross-talk between FXR and TGR5 signaling.

The selective activation of FXR, using OCA, was shown to reduce VAT mass, while improving insulin signaling in rPADs. However, our findings indicate that INT-767 is more potent than OCA on preadipocytes, not only improving their insulin sensitivity but also promoting browning differentiation.

Our results also point toward positive systemic effects of INT-767 treatment in MetS rabbits. Accordingly, NASH features were markedly reduced in HFD rabbits treated with INT-767, showing significantly decreased hepatic inflammation, steatosis, fibrosis and hypoxia. INT-767 effectively modulated the mRNA expression of several mediators of hepatic cholesterol uptake and metabolism and lipid droplet-associated genes. Overall, these data indicate potential molecular mechanisms underlying the protective effect of INT-767 from hepatic lipotoxicity. INT-767 treatment also induced hepatic expression of genes related to mitochondria biogenesis and function, and increased expression of PPARA. This finding is relevant, because the coordinated interplay between FXR and PPARA controls key hepatic metabolic pathways, including fatty acid and glucose metabolism (Preidis et al. 2017).

Hepatic steatosis per se could trigger the development of hepatic insulin resistance and inflammation (Petersen \& Shulman 2017). Supporting this view, INT-767 increased translocation to the plasma membrane of the insulindependent GLUT4, accompanied by upregulation of IRS1 and STAMP2, whose overexpression improves hepatic steatosis and insulin resistance in NAFLD (Kim et al. 2015). Consistent with previous results obtained in a mouse model of NASH (McMahan et al. 2013), INT-767 also counteracts HFD-induced liver inflammation as testified by a shift toward an anti-inflammatory macrophage M2 phenotype, which also promotes hepatic fibrosis regression (Krenkel \& Tacke 2017, Tacke 2017). In addition, INT-767 treatment reduces liver fibrosis and down-regulates mRNA expression of $S N A I 2$, a key profibrotic transcription factor (Wynn \& Ramalingam 2012).

Confirming the results obtained in VAT, analysis of the expression of FXR- and TGR5-related genes in the liver indicates that INT-767 behaves as a dual FXR/TGR5 agonist. INT-767 increased the hepatic expression of canonical TGR5 and FXR target genes, while decreasing CYP7A1 expression. In addition, INT-767 treatment stimulates the FXR-dependent FGF19 signaling not only in the liver but also in the intestine. Interestingly, INT-767 also upregulates the intestinal expression of the TGR5dependent PCSK1, an enzyme involved in the biosynthetic processing of GLP-1 (Dhanvantari et al. 2001). Therefore, the relative contribution of FXR and TGR5 activation via the small intestine on the general improvement of HFDinduced metabolic alterations could not be excluded. Further studies aimed at investigating more deeply the specific actions of FXR/TGR5 agonists on FGF19 signaling are most certainly warranted.

In summary, our study demonstrates that INT-767, a dual FXR and TGR5 agonist, reverses insulin resistance, hypercholesterolemia, VAT dysfunction and NASH, protecting rabbits from HFD-induced MetS. These beneficial effects of INT-767 were due, at least in part, to a direct effect on differentiating preadipocytes. Our data point toward rPADs metabolic rewiring promoted by INT-767, mainly through an FXR-dependent increase of insulin sensitivity and a TGR5-dependent improvement of mitochondrial function and browning differentiation. Indeed, INT-767, as a dual and potent agonist for FXR and TGR5, is able to induce beneficial effects related to both the single receptors. However, other non-mutually exclusive mechanisms may cooperate to improve the 
metabolic phenotype. Activation of FXR and TGR5 in the liver could, per se, explain the protective effects against NASH exerted by INT-767 treatment. Additionally, the effects of INT-767 on the FXR-dependent FGF19 and/or the TGR5-dependent proconvertase in intestine raise the obvious possibility that one or both these intestinal factors may contribute to the improved metabolic phenotype.

In conclusion, we provide evidence that INT-767 exerts remarkable pleiotropic effects in MetS, reflected by improved insulin sensitivity, enhanced mitochondrial function, inhibition of NASH development and amelioration of adipose tissue functions, leading to preadipocyte differentiation toward a metabolically healthy phenotype.

\section{Supplementary data}

This is linked to the online version of the paper at https://doi.org/10.1530/ JOE-17-0557.

\section{Declaration of interest}

P C, I C, T M, S F, E M, F C, C C, E S, A M, E R, D B, D G, B M, G B V, A G, M $M$ and $L V$ have no conflicts of interest. $L A$ is a scientific consultant for Intercept Pharmaceuticals.

\section{Funding}

This work was supported by Intercept Pharmaceuticals.

\section{References}

Adorini L, Pruzanski M \& Shapiro D 2012 Farnesoid X receptor targeting to treat nonalcoholic steatohepatitis. Drug Discovery Today 17 988-997. (https://doi.org/10.1016/j.drudis.2012.05.012)

Aon MA, Bhatt N \& Cortassa S 2014 Mitochondrial \& cellular mechanisms for managing lipid excess. Frontiers in Physiology 31282. (https://doi.org/10.3389/fphys.2014.00282)

Bach D, Pich S, Soriano FX Vega, N Baumgartner B, Oriola J, Daugaard JR, Lloberas J, Camps M, Zierath JR, et al. 2003 Mitofusin-2 determines mitochondrial network architecture and mitochondrial metabolism. A novel regulatory mechanism altered in obesity. Journal of Biological Chemistry 278 17190-17197. (https://doi.org/10.1074/jbc. M212754200)

Baglioni S, Francalanci M, Squecco R, Lombardi A, Cantini G, Angeli R, Gelmini S, Guasti D, Benvenuti S, Annunziato F, et al. 2009 Characterization of human adult stem-cell populations isolated from visceral and subcutaneous adipose tissue. FASEB Journal 10 3494-3505. (https://doi.org/10.1096/fj.08-126946)

Bartelt A, Widenmaier SB, Schlein C, Johann K, Goncalves RLS, Eguchi K, Fischer AW, Parlakgül G, Snyder NA, Nguyen TB, et al. 2018 Brown adipose tissue thermogenic adaptation requires Nrf1-mediated proteasomal activity. Nature Medicine 24 292-303. (https://doi. org/10.1038/nm.4481)

Bedossa P, Tordjman J, Aron-Wisnewsky J, Poitou C, Oppert JM, Torcivia A, Bouillot JL, Paradis V, Ratziu V \& Clément K 2016
Systematic review of bariatric surgery liver biopsies clarifies the natural history of liver disease in patients with severe obesity. Gut 66 1688-1696. (https://doi.org/10.1136/gutjnl-2016-312238)

Broeders EP, Nascimento EB, Havekes B, Brans B, Roumans KH, Tailleux A, Schaart G, Kouach M, Charton J, Deprez B, et al. 2015 The bile acid chenodeoxycholic acid increases human brown adipose tissue activity. Cell Metabolism 22 418-426. (https://doi.org/10.1016/j. cmet.2015.07.002)

Carino A, Cipriani S, Marchianò S, Biagioli M, Santorelli C, Donini A, Zampella A, Monti MC \& Fiorucci S 2017 BAR502, a dual FXR and GPBAR1 agonist, promotes browning of white adipose tissue and reverses liver steatosis and fibrosis. Scientific Reports 7 42801. (https:// doi.org/10.1038/srep42801)

Comeglio P, Filippi S, Sarchielli E, Morelli A, Cellai I, Corcetto F, Corno C, Maneschi E, Pini A, Adorini L, et al. 2017 Anti-fibrotic effects of chronic treatment with the selective FXR agonist obeticholic acid in the bleomycin-induced rat model of pulmonary fibrosis. Journal of Steroid Biochemistry and Molecular Biology 168 26-37. (https://doi. org/10.1016/j.jsbmb.2017.01.010)

Corona G, Mannucci E, Petrone L, Schulman C, Balercia G, Fisher AD, Chiarini V, Forti G \& Maggi M 2007 A comparison of NCEP-ATPIII and IDF metabolic syndrome definitions with relation to metabolic syndrome-associated sexual dysfunction. Journal of Sexual Medicine 4 789-796. (https://doi.org/10.1111/j.1743-6109.2007.00498.x)

DeLean A, Munson PJ \& Rodbard D 1978 Simultaneous analysis of families of sigmoidal curves: application to bioassay, radioligand assay, and physiological dose-response curves. American Journal of Physiology 235 E97-E102. (https://doi.org/10.1152/ ajpcell.1978.235.3.C97)

Dhanvantari S, Izzo A, Jansen E \& Brubaker PL 2001 Coregulation of glucagon-like peptide- 1 synthesis with proglucagon and prohormone convertase 1 gene expression in enteroendocrine GLUTag cells. Endocrinology 142 37-42. (https://doi.org/10.1210/endo.142.1.7870)

Fang S, Suh JM, Reilly SM, Yu E, Osborn O, Lackey D, Yoshihara E, Perino A, Jacinto S, Lukasheva Y, et al. 2015 Intestinal FXR agonism promotes adipose tissue browning and reduces obesity and insulin resistance. Nature Medicine 21 159-165. (https://doi.org/10.1038/ $\mathrm{nm} .3760)$

Filippi S, Vignozzi L, Morelli A, Chavalmane AK, Sarchielli E, Fibbi B, Saad F, Sandner P, Ruggiano P, Vannelli GB, et al. 2009 Testosterone partially ameliorates metabolic profile and erectile responsiveness to PDE5 inhibitors in an animal model of male metabolic syndrome. Journal of Sexual Medicine 6 3274-3288. (https://doi.org/10.1111/ j.1743-6109.2009.01467.x)

Fromme T, Kleigrewe K, Dunkel A, Retzler A, Li Y, Maurer S, Fischer N, Diezko R, Kanzleiter T, Hirschberg V, et al. 2018 Degradation of brown adipocyte purine nucleotides regulates uncoupling protein 1 activity. Molecular Metabolism 8 77-85. (https://doi.org/10.1016/j. molmet.2017.12.010)

Gao CL, Zhu C, Zhao YP, Chen XH, Ji CB, Zhang CM, Zhu JG, Xia ZK, Tong ML \& Guo XR 2010 Mitochondrial dysfunction is induced by high levels of glucose and free fatty acids in 3T3-L1 adipocytes. Molecular and Cellular Endocrinology 320 25-33. (https://doi. org/10.1016/j.mce.2010.01.039)

Goto T, Lee JY, Teraminami A, Kim YI, Hirai S, Uemura T, Inoue H, Takahashi N \& Kawada T 2011 Activation of peroxisome proliferatoractivated receptor-alpha stimulates both differentiation and fatty acid oxidation in adipocytes. Journal of Lipid Research 52 873-884. (https:// doi.org/10.1194/jlr.M011320)

Govers R 2014 Molecular mechanisms of GLUT4 regulation in adipocytes. Diabetes and Metabolism 40 400-410. (https://doi.org/10.1016/j. diabet.2014.01.005)

Haas B, Mayer P, Jennissen K, Scholz D, Berriel Diaz M, Bloch W, Herzig S, Fässler R \& Pfeifer A 2009 Protein kinase G controls brown fat cell differentiation and mitochondrial biogenesis. Science Signaling 2 ra78.

Hageman J, Herrema H, Groen AK \& Kuipers F 2010 A role of the bile salt receptor FXR in atherosclerosis. Arteriosclerosis, Thrombosis, 
and Vascular Biology 30 1519-1528. (https://doi.org/10.1161/ ATVBAHA.109.197897)

He F, Li J, Mu Y, Kuruba R, Ma Z, Wilson A, Alber S, Jiang Y, Stevens T, Watkins S, et al. 2006 Downregulation of endothelin-1 by farnesoid X receptor in vascular endothelial cells. Circulation Research 98 192-199. (https://doi.org/10.1161/01.RES.0000200400.55539.85)

Hoffmann LS, Etzrodt J, Willkomm L, Sanyal A, Scheja L, Fischer AW, Stasch JP, Bloch W, Friebe A, Heeren J, et al. 2015 Stimulation of soluble guanylyl cyclase protects against obesity by recruiting brown adipose tissue. Nature Communications 6 7235. (https://doi. org/10.1038/ncomms8235)

Houten SM, Watanabe M \& Auwerx J 2006 Endocrine functions of bile acids. EMBO Journal 25 1419-1425. (https://doi.org/10.1038/ sj.emboj.7601049)

Insel PA \& Ostrom RS 2003 Forskolin as a tool for examining adenylyl cyclase expression, regulation, and $\mathrm{G}$ protein signaling. Cellular and Molecular Neurobiology 23 305-314. (https://doi. org/10.1023/A:1023684503883)

Kanda T, Wakino S, Homma K, Yoshioka K, Tatematsu S, Hasegawa K, Takamatsu I, Sugano N, Hayashi K \& Saruta T 2006 Rho-kinase as a molecular target for insulin resistance and hypertension. FASEB Journal 20 169-171 (https://doi.org/10.1096/fj.05-4197fje)

Kawano Y \& Honda A 2017 Gene targeting in rabbits: single-step generation of knock-out rabbits by microinjection of CRISPR/Cas9 plasmids. Methods in Molecular Biology 1630 109-120.

Kelley DE, He J, Menshikova EV \& Ritov VB 2002 Dysfunction of mitochondria in human skeletal muscle in type 2 diabetes. Diabetes 51 2944-2950. (https://doi.org/10.2337/diabetes.51.10.2944)

Kim HY, Park SY, Lee MH, Rho JH, Oh YJ, Jung HU, Yoo SH, Jeong NY, Lee HJ, Suh S, et al. 2015 Hepatic STAMP2 alleviates high fat dietinduced hepatic steatosis and insulin resistance. Journal of Hepatology 63 477-485. (https://doi.org/10.1016/j.jhep.2015.01.025)

Klingenspor M, Bast A, Bolze F, Li Y, Maurer S, Schweizer S, Willershäuser M \& Fromme T 2017 Brown adipose tissue. In Adipose Tissue Biology, 2nd ed., pp 91-147. Ed ME Symonds. New York, NY, USA: Springer

Krampera M, Glennie S, Dyson J, Scott D, Laylor R, Simpson E \& Dazzi F 2003 Bone marrow mesenchymal stem cells inhibit the response of naive and memory antigenspecific $\mathrm{T}$ cells to their cognate peptide. Blood 101 3722-3729. (https://doi.org/10.1182/blood-2002-07-2104)

Krenkel O \& Tacke F 2017 Liver macrophages in tissue homeostasis and disease. Nature Reviews Immunology 17 306-321. (https://doi. org/10.1038/nri.2017.11)

Lefebvre P, Cariou B, Lien F, Kuipers F \& Staels B 2009 Role of bile acids and bile acid receptors in metabolic regulation. Physiological Reviews 89 147-191. (https://doi.org/10.1152/physrev.00010.2008)

Li J, Wilson A, Gao X, Kuruba R, Liu Y, Poloyac S, Pitt B, Xie W \& Li S 2009 Coordinated regulation of dimethylarginine dimethylaminohydrolase-1 and cationic amino acid transporter- 1 by farnesoid $\mathrm{X}$ receptor in mouse liver and kidney and its implication in the control of blood levels of asymmetric dimethylarginine. Journal of Pharmacology and Experimental Therapeutics 331 234-243. (https://doi.org/10.1124/jpet.109.153510)

Liesa M \& Shirihai OS 2013 Mitochondrial dynamics in the regulation of nutrientutilization and energy expenditure. Cell Metabolism 17 491-506. (https://doi.org/10.1016/j.cmet.2013.03.002)

Livak KJ \& Schmittgen TD 2001 Analysis of relative gene expression data using real-time quantitative PCR and the 2(-delta delta $\mathrm{C}(\mathrm{T})$ ) method. Methods 25 402-408. (https://doi.org/10.1006/meth.2001.1262)

Maneschi E, Morelli A, Filippi S, Cellai I, Comeglio P, Mazzanti B, Mello T, Calcagno A, Sarchielli E, Vignozzi L, et al. 2012 Testosterone treatment improves metabolic syndrome-induced adipose tissue derangements. Journal of Endocrinology 215 347-362. (https://doi. org/10.1530/JOE-12-0333)

Maneschi E, Vignozzi L, Morelli A, Mello T, Filippi S, Cellai I, Comeglio P, Sarchielli E, Calcagno A, Mazzanti B, et al. 2013 FXR activation normalizes insulin sensitivity in visceral preadipocytes of a rabbit model of MetS. Journal of Endocrinology 218 215-231. (https://doi. org/10.1530/JOE-13-0109)

Maneschi E, Cellai I, Aversa A, Mello T, Filippi S, Comeglio P, Bani D, Guasti D, Sarchielli E, Salvatore G, et al. 2016 Tadalafil reduces visceral adipose tissue accumulation by promoting preadipocytes differentiation towards a metabolically healthy phenotype: studies in rabbits. Molecular and Cellular Endocrinology 424 50-70. (https://doi. org/10.1016/j.mce.2016.01.015)

Mason RR \& Watt MJ 2015 Unraveling the roles of PLIN5: linking cell biology to physiology. Trends in Endocrinology and Metabolism 26 144-152. (https://doi.org/10.1016/j.tem.2015.01.005)

McMahan RH, Wang XX, Cheng LL, Krisko T, Smith M, El Kasmi K, Pruzanski M, Adorini L, Golden-Mason L, Levi M, et al. 2013 Bile acid receptor activation modulates hepatic monocyte activity and improves nonalcoholic fatty liver disease. Journal of Biological Chemistry 288 11761-11770. (https://doi.org/10.1074/jbc. M112.446575)

Mitschke MM, Hoffmann LS, Gnad T, Scholz D, Kruithoff K, Mayer P, Haas B, Sassmann A, Pfeifer A \& Kilic A 2013 Increased cGMP promotes healthy expansion and browning of white adipose tissue. FASEB Journal 27 1621-1630. (https://doi.org/10.1096/fj.12221580)

Miyazaki-Anzai S, Levi M, Kratzer A, Ting TC, Lewis LB \& Miyazaki M 2010 Farnesoid X receptor activation prevents the development of vascular calcification in ApoE-/- mice with chronic kidney disease. Circulation Research 106 1807-1817. (https://doi.org/10.1161/ CIRCRESAHA.109.212969)

Morelli A, Comeglio P, Filippi S, Sarchielli E, Cellai I, Vignozzi L, YehielyCohen R, Maneschi E, Gacci M, Carini M, et al. 2012 Testosterone and farnesoid X receptor agonist INT-747 counteract high fat diet-induced bladder alterations in a rabbit model of metabolic syndrome. Journal of Steroid Biochemistry and Molecular Biology 132 80-92. (https://doi. org/10.1016/j.jsbmb.2012.02.007)

Mudaliar S, Henry RR, Sanyal AJ, Morrow L, Marschall HU, Kipnes M, Adorini L, Sciacca CI, Clopton P, Castelloe E, et al. 2013 Efficacy and safety of the farnesoid $\mathrm{X}$ receptor agonist obeticholic acid in patients with type 2 diabetes and nonalcoholic fatty liver disease. Gastroenterology 145 574-582. (https://doi.org/10.1053/j. gastro.2013.05.042)

Neuschwander-Tetri BA, Loomba R, Sanyal AJ, Lavine JE, Van Natta ML, Abdelmalek MF, Chalasani N, Dasarathy S, Diehl AM, Hameed B, et al. 2015 Farnesoid X nuclear receptor ligand obeticholic acid for non-cirrhotic, non-alcoholic steatohepatitis (FLINT): a multicentre, randomised, placebo-controlled trial. Lancet 385 956-965. (https:// doi.org/10.1016/S0140-6736(14)61933-4)

Newmeyer DD \& Ferguson-Miller S 2003 Mitochondria: releasing power for life and unleashing the machineries of death. Cell 112 481-490. (https://doi.org/10.1016/S0092-8674(03)00116-8)

Nguyen TB, Louie SM, Daniele JR, Tran Q, Dillin A, Zoncu R, Nomura DK \& Olzmann JA 2017 DGAT1-dependent lipid droplet biogenesis protects mitochondrial function during starvation-induced autophagy. Developmental Cell 42 9-21. (https://doi.org/10.1016/j. devcel.2017.06.003)

Pathak P, Liu H, Boehme S, Xie C, Krausz KW, Gonzalez F \& Chiang JYL 2017 Farnesoid X receptor induces Takeda G-protein receptor 5 crosstalk to regulate bile acid synthesis and hepatic metabolism. Journal of Biological Chemistry 292 11055-11069. (https://doi.org/10.1074/jbc. M117.784322)

Pellicciari R, Gioiello A, Macchiarulo A, Thomas C, Rosatelli E, Natalini B, Sardella R, Pruzanski M, Roda A, Pastorini E, et al. 2009 Discovery of 6alpha-ethyl-23(S)-methylcholic acid (S-EMCA, INT-777) as a potent and selective agonist for the TGR5 receptor, a novel target for diabesity. Journal of Medicinal Chemistry 52 7958-7961. (https://doi. org/10.1021/jm901390p)
๑) 2018 Society for Endocrinology Published by Bioscientifica Ltd. Printed in Great Britain 
Petersen MC \& Shulman GI 2017 Roles of diacylglycerols and ceramides in hepatic insulin resistance. Trends in Pharmacological Sciences $\mathbf{3 8}$ 649-665. (https://doi.org/10.1016/j.tips.2017.04.004)

Preidis GA, Kim KH \& Moore DD 2017 Nutrient-sensing nuclear receptors PPAR $\alpha$ and FXR control liver energy balance. Journal of Clinical Investigation 127 1193-1201. (https://doi.org/10.1172/JCI88893)

Rapizzi E, Fucci R, Giannoni E, Canu L, Richter S, Cirri P \& Mannelli M 2015 Role of microenvironment on neuroblastoma SK-N-AS SDHBsilenced cell metabolism and function. Endocrine-Related Cancer 22 409-417. (https://doi.org/10.1530/ERC-14-0479)

Rizzo G, Passeri D, De Franco F, Ciaccioli G, Donadio L, Rizzo G, Orlandi S, Sadeghpour B, Wang XX, Jiang T, et al. 2010 Functional characterization of the semisynthetic bile acid derivative INT-767, a dual farnesoid X receptor and TGR5 agonist. Molecular Pharmacology 78 617-630. (https://doi.org/10.1124/mol.110.064501)

Rousset S, Alves-Guerra MC, Mozo J, Miroux B, Cassard-Doulcier AM, Bouillaud F \& Ricquier D 2004 The biology of mitochondrial uncoupling proteins. Diabetes 53 S130-S135. (https://doi. org/10.2337/diabetes.53.2007.S130)

Schindelin J, Arganda-Carreras I, Frise E, Kaynig V, Longair M, Pietzsch T, Preibisch S, Rueden C, Saalfeld S, Schmid B, et al. 2012 Fiji: an open-source platform for biological-image analysis. Nature Methods 9 676-682. (https://doi.org/10.1038/nmeth.2019)

Shang Q, Guo GL, Honda A, Shi D, Saumoy M, Salen G \& Xu G 2014 Bile acid flux through portal but not peripheral veins inhibits CYP7A1 expression without involvement of ileal FGF19 in rabbits. American Journal of Physiology: Gastrointestinal and Liver Physiology $\mathbf{3 0 7}$ G479-G486. (https://doi.org/10.1152/ajpgi.00062.2014)

Student AK, Hsu RY \& Lane MD 1980 Induction of fatty acid synthetase synthesis in differentiating 3T3-L1 preadipocytes. Journal of Biological Chemistry 255 4745-4750.

Tacke F 2017 Targeting hepatic macrophages to treat liver diseases. Journal of Hepatology 66 1300-1312. (https://doi.org/10.1016/j. jhep.2017.02.026)

Thomas C, Pellicciari R, Pruzanski M, Auwerx J \& Schoonjans K 2008 Targeting bile-acid signalling for metabolic diseases. Nature Reviews Drug Discovery 7 678-693. (https://doi.org/10.1038/nrd2619)

Thomas C, Gioiello A, Noriega L, Strehle A, Oury J, Rizzo G, Macchiarulo A, Yamamoto H, Mataki C, Pruzanski M, et al. 2009 TGR5-mediated bile acid sensing controls glucose homeostasis. Cell Metabolism 10 167-177. (https://doi.org/10.1016/j.cmet.2009.08.001)

Toledo FG, Watkins S \& Kelley DE 2006 Changes induced by physical activity and weight loss in the morphology of intermyofibrillar mitochondria in obese men and women. Journal of Clinical
Endocrinology and Metabolism 91 3224-3227. (https://doi.org/10.1210/ jc.2006-0002)

Vignozzi L, Filippi S, Comeglio P, Cellai I, Sarchielli E, Morelli A, Rastrelli G, Maneschi E, Galli A, Vannelli GB, et al. 2014 Nonalcoholic steatohepatitis as a novel player in metabolic syndrome-induced erectile dysfunction: an experimental study in the rabbit. Molecular and Cellular Endocrinology 384 143-154. (https://doi.org/10.1016/j. mce.2014.01.014)

Vignozzi L, Morelli A, Cellai I, Filippi S, Comeglio P, Sarchielli E, Maneschi E, Vannelli GB, Adorini L \& Maggi M 2017 Cardiopulmonary protective effects of the selective FXR agonist obeticholic acid in the rat model of monocrotaline-induced pulmonary hypertension. Journal of Steroid Biochemistry and Molecular Biology 165 277-292. (https://doi.org/10.1016/j.jsbmb.2016.07.004)

Wang XX, Luo Y, Wang D, Adorini L, Pruzanski M, Dobrinskikh E \& Levi M 2017 A dual agonist of farnesoid X receptor (FXR) and the G protein-coupled receptor TGR5, INT-767, reverses age-related kidney disease in mice. Journal of Biological Chemistry 292 12018-12024. (https://doi.org/10.1074/jbc.C117.794982)

Watanabe M, Houten SM, Mataki C, Christoffolete MA, Kim BW, Sato H, Messaddeq N, Harney JW, Ezaki O, Kodama T, et al. 2006 Bile acids induce energy expenditure by promoting intracellular thyroid hormone activation. Nature 439 484-489. (https://doi.org/10.1038/ nature04330)

Wellen KE, Fucho R, Gregor MF, Furuhashi M, Morgan C, Lindstad T, Vaillancourt E, Gorgun CZ, Saatcioglu F \& Hotamisligil GS 2007 Coordinated regulation of nutrient and inflammatory responses by STAMP2 is essential for metabolic homeostasis. Cell 129 537-548. (https://doi.org/10.1016/j.cell.2007.02.049)

Wynn TA \& Ramalingam TR 2012 Mechanisms of fibrosis: therapeutic translation for fibrotic disease. Nature Medicine 18 1028-1040. (https://doi.org/10.1038/nm.2807)

Zannettino AC, Paton S, Arthur A, Khor F, Itescu S, Gimble JM \& Gronthos S 2008 Multipotential human adipose-derived stromal stem cells exhibit a perivascular phenotype in vitro and in vivo. Journal of Cellular Physiology 214 413-421. (https://doi.org/10.1002/jcp.21210)

Zhang Y, Lee FY, Barrera G, Lee H, Vales C, Gonzalez FJ, Willson TM \& Edwards PA 2006 Activation of the nuclear receptor FXR improves hyperglycemia and hyperlipidemia in diabetic mice. PNAS 103 1006-1011. (https://doi.org/10.1073/pnas.0506982103)

Zuk PA, Zhu M, Mizuno H, Huang J, Futrell JW, Katz AJ, Benhaim P, Lorenz HP \& Hedrick MH 2001 Multilineage cells from human adipose tissue: implications for cell-based therapies. Tissue Engineering 7 211-228. (https://doi.org/10.1089/107632701300062859)
Received in final form 16 May 2018

Accepted 29 May 2018
() 2018 Society for Endocrinology Published by Bioscientifica Ltd. Printed in Great Britain 